Rigidity theory for matroids (with Mike Develin and Victor Reiner) Comment. Math. Helv. 82 (2007), 197--233. Publisher's official version: http://dx.doi.org/10.4171/CMH/89, Open Access version: http://kuscholarworks.ku.edu/dspace/.

[This document contains the author's accepted manuscript. For the publisher's version, see the link in the header of this document.]

\title{
RIGIDITY THEORY FOR MATROIDS
}

\author{
MIKE DEVELIN, JEREMY L. MARTIN AND VICTOR REINER
}

\begin{abstract}
Combinatorial rigidity theory seeks to describe the rigidity or flexibility of bar-joint frameworks in $\mathbb{R}^{d}$ in terms of the structure of the underlying graph $G$. The goal of this article is to broaden the foundations of combinatorial rigidity theory by replacing $G$ with an arbitrary representable matroid $M$. The ideas of rigidity independence and parallel independence, as well as Laman's and Recski's combinatorial characterizations of 2-dimensional rigidity for graphs, can naturally be extended to this wider setting. As we explain, many of these fundamental concepts really depend only on the matroid associated with $G$ (or its Tutte polynomial), and have little to do with the special nature of graphic matroids or the field $\mathbb{R}$.

Our main result is a "nesting theorem" relating the various kinds of independence. Immediate corollaries include generalizations of Laman's Theorem, as well as the equality of 2-rigidity and 2-parallel independence. A key tool in our study is the space of photos of $M$, a natural algebraic variety whose irreducibility is closely related to the notions of rigidity independence and parallel independence.

The number of points on this variety, when working over a finite field, turns out to be an interesting Tutte polynomial evaluation.
\end{abstract}

\section{CONTENTS}

1. Introduction: a brief tour through rigidity theory

2. Main definitions: from graphs to matroids

3. Laman independence

3.1. When is the Laman complex matroidal?

3.2. Equivalent characterizations

3.3. Digression on polymatroids

4. Slope independence and the space of photos

5. Counting photos

6. Rigidity and parallel independence

6.1. Interpreting $\mathcal{R}^{d}(M)$ and $\mathcal{H}^{d}(M)$

6.2. The Nesting Theorem

7. Examples: Uniform matroids

8. More on $\mathcal{R}^{d}(M)$ : invariance and stabilization 20

9. Open problems 23

Acknowledgments 23

References

\section{Introduction: A BRIEF TOUR THROUGH RIGIDITY THEORY}

Combinatorial rigidity theory is concerned with frameworks built out of bars and joints in $\mathbb{R}^{d}$, representing the vertices $V$ and edges $E$ of an (undirected, finite) graph $G$. (For comprehensive treatments of the subject, see, e.g., $[4,19,20]$.) The motivating problem is to determine how the combinatorics of $G$ governs the rigidity

Date: November 30, 2005.

1991 Mathematics Subject Classification. 05B35, 52C25, 14N20.

Key words and phrases. matroid, combinatorial rigidity, parallel redrawing, Laman's Theorem, Tutte polynomial.

First author supported by the American Institute of Mathematics. Second author partially supported by an NSF Postdoctoral Fellowship. Third author partially supported by NSF grant DMS-0245379. 
or flexibility of its frameworks. Typically, one makes a generic choice of coordinates

$$
p=\left\{p_{v}: v \in V\right\} \subset \mathbb{R}^{d}
$$

for the vertices of $G$, and considers infinitesimal motions $\Delta p$ of the vertices. The following two questions are pivotal:

(I.) What is the dimension of the space of infinitesimal motions $\Delta p$ that preserve all the (squared) edge lengths $Q\left(p_{u}-p_{v}\right)$, for $\{u, v\} \in E$, where $Q(x)=\sum_{i=1}^{d} x_{i}^{2}$ ?

(II.) What is the dimension of the space of infinitesimal motions $\Delta p$ that preserve all the edge directions $p_{u}-p_{v}$ regarded as slopes, that is, up to scaling?

The answers to these questions are known to be determined by certain linear dependence matroids represented over transcendental extensions of $\mathbb{R}$, as we now explain.

First, the $d$-dimensional rigidity matroid $\mathcal{R}^{d}(G)$ is the matroid represented by the vectors

$$
\left\{\left(e_{u}-e_{v}\right) \otimes\left(p_{u}-p_{v}\right):\{u, v\} \in E\right\}
$$

lying in $\mathbb{R}^{|V|} \otimes \mathbb{R}(p)^{d}$, where $\mathbb{R}(p)$ is the extension of $\mathbb{R}$ by a collection of $d|V|$ transcendentals $p$, thought of as the coordinates of a generic embedding as in (1). The $|E| \times d|V|$ rigidity matrix $R^{d}(G)$ has as its rows the $|E|$ vectors in (2). Then the nullspace of $R^{d}(G)$ is the space of infinitesimal motions of the vertices that preserve edge distances (because $R^{d}(G)$ is $\frac{1}{2}$ times the Jacobian in the variables $p$ of the vector of squared edge lengths $Q\left(p_{u}-p_{v}\right)$; cf. Remark 6.2 below). Since row rank equals column rank, knowing the matroid $\mathcal{R}^{d}(G)$ represented by the rows of $R^{d}(G)$ answers question (I).

Second, the $d$-dimensional parallel matroid $\mathcal{P}^{d}(G)$ is the matroid represented by the vectors

$$
\left\{\left(e_{u}-e_{v}\right) \otimes \eta_{u, v}^{(j)}:\{u, v\} \in E, j=1,2, \ldots, d-1\right\}
$$

where for each edge $\{u, v\} \in E$, the vectors $\eta_{u, v}^{(1)}, \ldots, \eta_{u, v}^{(d-1)}$ are generically chosen normals to $p_{u}-p_{v}$ in $\mathbb{R}^{d}$. Again, we should consider the vectors in (3) as lying in $\mathbb{R}^{|V|} \otimes \mathbb{R}(p, \eta)^{d}$, where $\mathbb{R}(p, \eta)$ is an extension of $\mathbb{R}$ by $d|V|$ transcendentals $p$ and $(d-1)|E|$ transcendentals $\eta$. In analogy to the preceding paragraph, the $|E| \times d|V|$ parallel matrix $P^{d}(G)$ has as its rows the $|E|$ vectors in (3), and its nullspace is the space of infinitesimal motions of the vertices that preserve all edge directions. Consequently, the matroid $\mathcal{P}^{d}(G)$ represented by the rows of $P^{d}(G)$ provides the answer to question (II).

Some features of the theory are as follows:

- For $d=1$, the rigidity matroid coincides with the usual graphic matroid for $G$ (while the parallel matroid is a trivial object).

- For $d=2$, the rigidity and parallel matroids coincide [19, Corollary 4.1.3]. Furthermore, this matroid $\mathcal{R}^{2}(G)=\mathcal{P}^{2}(G)$ has many equivalent combinatorial reformulations, of which the best known is Laman's condition [6]: $A \subseteq E$ is 2-rigidity-independent if and only if for every subset $A^{\prime} \subseteq A$

$$
\begin{aligned}
2\left|V\left(A^{\prime}\right)\right|-3 & \geq\left|A^{\prime}\right|, \quad \text { or equivalently } \\
2\left(\left|V\left(A^{\prime}\right)\right|-1\right) & >\left|A^{\prime}\right|
\end{aligned}
$$

where $V\left(A^{\prime}\right)$ denotes the set of vertices incident to at least one edge in $A^{\prime}$. We will refer to this coincidence between $\mathcal{R}^{2}(G), \mathcal{P}^{2}(G)$ and the matroid defined by Laman's condition as the planar trinity.

- For $d>2$, the parallel matroid has a simple combinatorial characterization that generalizes Laman's condition, while an analogous description for the rigidity matroid is not known.

\section{MAIN DEFINITIONS: FROM GRAPHS TO MATROIDS}

The purpose of this article is to broaden the scope of rigidity theory by replacing the graph $G$ with a more general object: a matroid $M$ represented over an arbitrary field $\mathbb{F}$. As we shall see, the notions of rigidity and parallel independence, as well as Laman's combinatorial characterization, admit natural generalizations to the setting of matroids.

In the process, we will see that many of the main results of do not depend on the special properties of graphs (or graphic matroids), nor on the field $\mathbb{R}$, but indeed remain valid for any matroid $M$ represented as above. In the process, we are led naturally to an algebraic variety called the space of $k$-plane-marked 
$d$-photos of $M$. Just as a bar-joint framework may be regarded as an embedding of a graph in $\mathbb{R}^{d}$, a photo of $M$ is a "model" of $M$ in $\mathbb{F}^{d}$.

Whether or not the photo space is irreducible plays a key role in characterizing the matroid analogues of rigidity independence and parallel independence. In turn, the question of irreducibility can be answered combinatorially, using some elementary algebraic geometry and the classic matroid partitioning result of Edmonds [3]. We note in addition that when the field $\mathbb{F}$ is finite, the number of photos of $M$ is counted by an evaluation of the Tutte polynomial using $q$-binomial coefficients.

In order to summarize our results, we define the main protagonists here. Recall that a simplicial complex on vertex set $E$ is a collection $\mathcal{I}$ of subsets of $E$ satisfying the following hereditary condition: if $I \in \mathcal{I}$ and $I^{\prime} \subseteq I$, then $I^{\prime} \in \mathcal{I}$. The independent sets of a matroid always form a simplicial complex. From here on we will make free use of standard terminology and notions from matroid theory; background and definitions may be found in standard texts such as $[1,12,17]$.

Definition 2.1 ( $m$-Laman independence, $m$-Laman complex). Let $E$ be a set of cardinality $n$, and let $M$ be a (not necessarily representable) matroid on ground set $E$, with rank function $r$. For $m$ a real number in the open interval $(1, \infty)_{\mathbb{R}}$, say that $A \subseteq E$ is $m$-Laman independent if

$$
m \cdot r\left(A^{\prime}\right)>\left|A^{\prime}\right| \quad \text { for all nonempty subsets } A^{\prime} \subseteq A .
$$

The $m$-Laman complex $\mathcal{L}^{m}(M)$ is defined as the abstract simplicial complex of all $m$-Laman independent subsets of $E$.

We will prove combinatorially that

- If $m$ is a positive integer, then $\mathcal{L}^{m}(M)$ is the collection of independent sets of a matroid (Theorem 3.1), but this is not true in general for other values of $m$.

- If $m$ is a positive integer, then $\mathcal{L}^{m}(M)$ has several other combinatorial characterizations (Theorem 3.6), including a generalization of Recski's Theorem.

- If $m=2$ and $M$ is representable, then the matroid $\mathcal{L}^{2}(M)$ coincides with the 2-dimensional rigidity and parallel matroids, defined below (Corollary 6.6).

Throughout the rest of the introduction, let $M$ be a represented matroid; that is, a matroid equipped with a representation over some field $\mathbb{F}$ by vectors $E=\left\{v_{1}, \ldots, v_{n}\right\} \subset \mathbb{F}^{r}$. It is worth emphasizing that we are not regarding $M$ as an abstract matroid; that is, the vectors $\left\{v_{1}, \ldots, v_{n}\right\}$ are part of the data of $M$. For notational convenience, we identify the ground set $E$ with the numbers $[n]:=\{1,2, \ldots, n\}$. Denote by $\mathbb{G} r\left(k, \mathbb{F}^{d}\right)$ the Grassmannian of $k$-planes in $\mathbb{F}^{d}$, regarded as a projective variety over $\mathbb{F}$ via the usual Plücker embedding into $\mathbb{P}_{\left(\begin{array}{l}d \\ k\end{array}\right)-1}$.

When $m>1$ is a rational number, the Laman complex $\mathcal{L}^{m}(M)$ is closely related to an algebraic variety that we now define.

Definition 2.2 (photo space, $(k, d)$-slope independence, $(k, d)$-slope complex). Let $M$ be a matroid equipped with representation $\left\{v_{1}, \ldots, v_{n}\right\}$ as above. The corresponding space of $k$-plane-marked d-photos (or just $(k, d)$-photos) is the algebraic set

$$
X_{k, d}(M):=\left\{\left(\varphi, W_{1}, \ldots, W_{n}\right) \in \operatorname{Hom}_{\mathbb{F}}\left(\mathbb{F}^{r}, \mathbb{F}^{d}\right) \times \mathbb{G} r\left(k, \mathbb{F}^{d}\right)^{n}: \varphi\left(v_{i}\right) \in W_{i} \text { for } i=1, \ldots, n\right\} .
$$

The photo space of a matroid is analogous to the picture space of a graph, as defined in $[7,8]$. One may think of the map $\varphi \in \operatorname{Hom}_{\mathbb{F}}\left(\mathbb{F}^{r}, \mathbb{F}^{d}\right)$ as projecting the vectors $\left\{v_{i}\right\}$ into a space $\mathbb{F}^{d}$ of dimension possibly less than $r$, like a camera taking a photo of the $\left\{v_{i}\right\}$ on photographic paper that looks like $\mathbb{F}^{d}$. The $k$-plane $W_{i}$ in $\mathbb{F}^{d}$ is thought of as a "marking" of the image vector $\varphi\left(v_{i}\right)$ in the photo, so that $W_{i}$ is constrained to contain $\phi\left(v_{i}\right)$. Of course, whenever $\varphi\left(v_{i}\right)=0$ (perhaps the camera $\varphi$ caught $v_{i}$ at a bad angle), this $k$-plane $W_{i}$ is unconstrained. The idea of $(k, d)$-slope independence is to measure how independently these marking $k$-planes can vary while obeying these constraints, when none of the $v_{i}$ are annihilated by $\varphi$. The linear dependences among the $v_{i}$ force linear dependences among their image vectors $\varphi\left(v_{i}\right)$, and hence algebraic constraints among the subspaces $W_{i}$.

Define a Zariski open subset of $X_{k, d}(M)$ (called the non-annihilating cellule; see Definition 4.1 below) by

$$
X_{k, d}^{\varnothing}(M):=\left\{\left(\varphi, W_{1}, \ldots, W_{n}\right) \in X_{k, d}(M): \varphi\left(v_{i}\right) \neq 0 \text { for } i=1,2, \ldots, n\right\}
$$


and consider its image under the projection map

$$
\operatorname{Hom}\left(\mathbb{F}^{r}, \mathbb{F}^{d}\right) \times \mathbb{G} r\left(k, \mathbb{F}^{d}\right)^{n} \stackrel{\pi}{\longrightarrow} \mathbb{G} r\left(k, \mathbb{F}^{d}\right)^{n} .
$$

This image measures the constraints on the $W_{i}$ when none of the $v_{i}$ are mapped to zero; specifically, we define $M$ to be $(k, d)$-slope independent if $\pi X_{k, d}^{\varnothing}(M)$ is Zariski dense in $\mathbb{G} r\left(k, \mathbb{F}^{d}\right)^{n}$. The $(k, d)$-slope complex is defined as

$$
\mathcal{S}^{k, d}(M):=\left\{A \subseteq E:\left.M\right|_{A} \text { is }(k, d) \text {-slope independent }\right\} .
$$

A third notion of matroid rigidity generalizes the $d$-dimensional rigidity matroid $\mathcal{R}^{d}(G)$ of a graph $G$.

Definition 2.3 (rigidity matroid, rigidity complex). Let $M$ be a matroid equipped with representation $\left\{v_{1}, \ldots, v_{n}\right\}$ as above, and let $d$ be a positive integer. The d-dimensional (generic) rigidity matroid is the matroid represented by the vectors

$$
\left\{v_{i} \otimes \varphi\left(v_{i}\right)\right\}_{i=1}^{n} \subset \mathbb{F}^{r} \otimes_{\mathbb{F}} \mathbb{F}(\varphi)^{d} .
$$

where $\mathbb{F}(\varphi)$ is the field extension of $\mathbb{F}$ by $d r$ transcendentals giving the entries of the matrix $\varphi: \mathbb{F}^{r} \rightarrow \mathbb{F}(\varphi)^{d}$. The $d$-rigidity complex $\mathcal{R}^{d}(M)$ is the complex of independent sets of the $d$-dimensional rigidity matroid, and the $d$-rigidity matrix $R^{d}(M)$ is the $n \times d r$ matrix whose rows are given by the vectors $(9)$.

To interpret this construction, consider the pseudo-distance quadratic form $Q(x):=\sum_{i=1}^{d} x_{i}^{2}$ on $\mathbb{F}(\varphi)^{d}$. Provided that the field $\mathbb{F}$ has characteristic $\neq 2$, one can interpret the nullspace of $R^{d}(M)$ as the space of infinitesimal changes of $\varphi$ that preserve the values $Q\left(\varphi\left(v_{i}\right)\right)$ for all $i=1,2, \ldots, n$. (See Proposition 6.1(ii).)

Definition 2.4 (hyperplane-marking matroid). Let $M$ be a matroid represented by $v_{1}, \ldots, v_{n} \in \mathbb{F}^{r}$ as above. Its (d-dimensional, generic) hyperplane-marking matroid is the matroid represented over $\mathbb{F}(\varphi, \eta)$ by the vectors

$$
\left\{v_{i} \otimes \eta_{i}\right\}_{i=1}^{n} \subset \mathbb{F}^{r} \otimes_{\mathbb{F}} \mathbb{F}(\varphi, \eta)^{d}
$$

where $\mathbb{F}(\varphi, \eta)$ is the extension of $\mathbb{F}$ by $d r$ transcendentals $\varphi_{i j}$ (the entries of the matrix $\left.\varphi\right)$ and $(d-1) n$ more transcendentals $\eta_{i j}$ (the coordinates of the vectors $\eta_{i}$ normal to $\varphi\left(v_{i}\right)$ ). The complex $\mathcal{H}^{d}(M)$ is defined to be the complex of independent sets of this matroid.

To interpret the notion of rigidity independence modeled by $\mathcal{H}^{d}(M)$, one should regard lack of rigidity as the ability to deform $\varphi$ so that the images $\varphi\left(v_{i}\right)$ of the ground set elements vary, but membership in their orthogonal complement hyperplanes is preserved. The most important instance of the hyperplane-marking matroid uses the $(d-1)$-parallel extension of $M$, the matroid $(d-1) M$ whose ground set consists of $d-1$ parallel copies of each element of $E$. The (d-dimensional, generic) parallel matroid is defined as

$$
\mathcal{P}^{d}(M):=\mathcal{H}^{d}((d-1) M),
$$

and the $d$-parallel matrix $P^{d}(M)$ is defined as the $n \times d r$ matrix whose rows represent $\mathcal{H}^{d}((d-1) M)$. Its nullspace consists of the infinitesimal changes $\Delta \varphi$ in the matrix $\varphi$ which preserve the slopes of all the direction vectors $\varphi\left(v_{i}\right)$ (see Proposition $6.1(\mathrm{i})$ ).

These definitions generalize the ordinary definitions from the rigidity theory of graphs. Strikingly, the geometric constraints on the photo space can be categorized combinatorially: the identity

$$
\mathcal{S}^{k, d}(M)=\mathcal{L}^{\frac{d}{d-k}}(M),
$$

(Corollary 4.4) provides a geometric interpretation of $\mathcal{L}^{m}(M)$ for rational $m$.

The slope complex $\mathcal{S}^{k, d}(M)$ is closely related to the rigidity and parallel matroids. The precise relationship is given by the Nesting Theorem (Theorem 6.5):

$$
\mathcal{S}^{1, d}(M) \subseteq \mathcal{R}^{d}(M) \subseteq \mathcal{L}^{d}(M)=\mathcal{H}^{d}(M)=\mathcal{S}^{d-1, d}(M)
$$

for all integers $d \geq 2$. In particular, when $d=2$,

$$
\mathcal{H}^{2}(M)=\mathcal{S}^{1,2}(M)=\mathcal{R}^{2}(M)=\mathcal{L}^{2}(M) .
$$

Thus matroid rigidity theory leads to a conceptual proof of the planar trinity (the second and third inequalities in (10)). 
For $d \geq 3$, the $d$-rigidity matroid $\mathcal{R}^{d}(M)$ is the hardest of these objects to understand (as it is for graphic matroids). One fundamental question is whether $\mathcal{R}^{d}(M)$ depends on the choice of representation of $M$. It is invariant for $d=2$ (by the Nesting Theorem) and up to projective equivalence of representations (Proposition 8.1), but the problem remains open for the general case. We also study the behavior of the $d$-rigidity matroid as $d \rightarrow \infty$, and show (Proposition 8.4) that $R^{d}(M)$ stabilizes when $d \geq r(M)$.

\section{LAMAN INDEPENDENCE}

The central result of this section, Theorem 3.1, states that the generalized Laman's condition (5) always gives a matroid when $m$ is an integer. The proof is completely combinatorial; that is, it is a statement about abstract matroids, not represented matroids. In addition, we describe some useful equivalent characterizations of $d$-Laman independence: one uses the Tutte polynomial, another is reminiscent of Recski's Theorem, and another is related to Edmonds' theorem on decomposing a matroid into independent sets.

\subsection{When is the Laman complex matroidal?}

Theorem 3.1. $\quad$ (i) Let $d$ be a positive integer and let $M$ be any matroid. Then the simplicial complex $\mathcal{L}^{d}(M)$ is a matroid complex.

(ii) Let $m \in(1, \infty)_{\mathbb{R}}$ be a real number which is not an integer. Then there exists a represented matroid $M$ for which $\mathcal{L}^{m}(M)$ is not a matroid complex.

Proof. For the first assertion, it is most convenient to use the characterization of matroids by circuit axioms [1, eq. 6.13, p. 264]. Define $\mathcal{C}$ to be the collection of those subsets of $E$ which are minimal among nonmembers of $\mathcal{L}^{d}(M)$. We wish to show that $\mathcal{C}$ satisfies the axioms for the circuits of a matroid. Since $\mathcal{L}^{d}(M)$ is a simplicial complex, we only need check the circuit exchange axiom:

if $C, C^{\prime} \in \mathcal{C}$ with $C \neq C^{\prime}$, and $e \in C \cap C^{\prime}$, then there exists $C^{\prime \prime} \in \mathcal{C}$ such that $C^{\prime \prime} \subseteq\left(C \cup C^{\prime}\right) \backslash\{e\}$.

Since $C, C^{\prime}$ are minimal among the sets not satisfying the hereditary property (5), we claim that

$$
\begin{aligned}
|C| & =d \cdot r(C), \\
\left|C^{\prime}\right| & =d \cdot r\left(C^{\prime}\right),
\end{aligned}
$$

where $r$ is the rank function of $M$. To see this claim, note that $|C| \geq d \cdot r(C)$, and if this inequality were strict, then

$$
|C-e| \geq d \cdot r(C) \geq d \cdot r(C-e)
$$

for any $e \in C$, contradicting the statement that $C$ is a minimal set not satisfying (5). Note also that $C \cap C^{\prime}$ is a proper subset of each of $C, C^{\prime}$ and hence

$$
\left|C \cap C^{\prime}\right|<d \cdot r\left(C \cap C^{\prime}\right) .
$$

Since $d$ is an integer, the last condition may be rewritten as

$$
\left|C \cap C^{\prime}\right|+1 \leq d \cdot r\left(C \cap C^{\prime}\right) .
$$

The rank submodular inequality $r\left(C \cup C^{\prime}\right) \leq r(C)+r\left(C^{\prime}\right)-r\left(C \cap C^{\prime}\right)$ then implies

$$
\begin{aligned}
d \cdot r\left(\left(C \cup C^{\prime}\right) \backslash\{e\}\right) & \leq d \cdot r\left(C \cup C^{\prime}\right) \\
& \leq d \cdot r(C)+d \cdot r\left(C^{\prime}\right)-d \cdot r\left(C \cap C^{\prime}\right) \\
& \leq|C|+\left|C^{\prime}\right|-\left|C \cap C^{\prime}\right|-1 \\
& =\left|\left(C \cup C^{\prime}\right) \backslash\{e\}\right| .
\end{aligned}
$$

So $\left(C \cup C^{\prime}\right) \backslash\{e\}$ is not in $\mathcal{L}^{d}(M)$, hence contains some element of $\mathcal{C}$. This establishes (i).

We now prove (ii). Suppose that $m \in(1, \infty)_{\mathbb{R}}$ is not an integer, and let $c:=\lfloor m\rfloor$ (the greatest integer $\leq m$ ). Choose positive integers $a, b$ satisfying the inequalities (11) in Lemma 3.2 below. We will explicitly construct a represented matroid $M_{a, b, c}$ such that $\mathcal{L}^{m}\left(M_{a, b, c}\right)$ is not a matroid complex.

Let $\mathbb{F}$ be a sufficiently large (for example, infinite) field, let $V$ be a $(2 b-1)$-dimensional vector space over $\mathbb{F}$, and let $V_{1}, V_{2}$ be two $b$-dimensional subspaces of $V$ whose intersection $V_{1} \cap V_{2}=\ell$ is a line. Let $X=\left\{x_{1}, \ldots, x_{c}\right\}$ be a set of $c$ nonzero vectors on $\ell$. For $i=1,2$, choose a set $Y_{i} \subseteq V_{i}$ of cardinality $a-c$ 
generically (this is always possible if $\mathbb{F}$ is sufficiently large). Note in particular that no member of $Y_{1} \cup Y_{2}$ lies on $\ell$.

Let $M_{a, b, c}$ be the matroid represented over $\mathbb{F}$ by $E=X \cup Y_{1} \cup Y_{2}$, and denote by $\mathcal{C}$ the set of subsets of $E$ that are minimal among nonmembers of $\mathcal{L}^{m}\left(M_{a, b, c}\right)$. We claim that $\mathcal{C}$ does not satisfy the circuit exchange axiom. To see this, let $C_{i}=X \cup Y_{i}$ for $i=1,2$ and observe that

$$
m \cdot r\left(C_{i}\right)=m b \leq a=\left|C_{i}\right|
$$

so $C_{i} \notin \mathcal{L}^{m}\left(M_{a, b, c}\right)$. In fact, we claim that $C_{i} \in \mathcal{C}$. Indeed, let $I$ be any nonempty proper subset of $C_{i}$ and let $J=I \cap Y_{i}$. Since $r(X)=1$, and by the generic choice of $Y_{1}$ and $Y_{2}$, we have

$$
\begin{aligned}
r(J) & =\min (|J|, b), \\
r(I) & =\min (|J|+1, b), \\
m \cdot r(I) & =\min (m|J|+m, m b) .
\end{aligned}
$$

Now Lemma 3.2 implies that $m b \geq a=\left|C_{i}\right|>|I|$. Since $m$ is not an integer, we have also

$$
m|J|+m>|J|+c=|J|+|X| \geq|I| .
$$

In all cases $m \cdot r(I)>|I|$. It follows that $C_{i} \in \mathcal{C}$.

Now, let $x_{i} \in X$, and let $I=\left(C_{1} \cup C_{2}\right) \backslash\left\{x_{i}\right\}=E \backslash\left\{x_{i}\right\}$. Then every nonempty subset $I^{\prime} \subseteq I$ satisfies (5). (We omit the routine but tedious calculation, which involves eight cases, depending on how $I^{\prime}$ meets each of $X, Y_{1}$ and $Y_{2}$.) That is, $I$ is $m$-Laman-independent, hence contains no element of $\mathcal{C}$. Therefore $\mathcal{C}$ fails the circuit exchange axiom, and we are done.

The following numerical lemma was used in the proof of Theorem 3.1.

Lemma 3.2. Let $m \in(1, \infty)_{\mathbb{R}}$ be a real number which is not an integer, and let $c:=\lfloor m\rfloor$. Then there exist positive integers $a, b$ such that

$$
\frac{a-1}{b}<\frac{2 a-c-1}{2 b-1}<m \leq \frac{a}{b}
$$

Proof. First, note that the third inequality implies the first one. Indeed, if $m \leq a / b$, then

$$
b+a \geq 1+a \geq 1+b m>1+b c,
$$

which implies in turn that $2 a b-a-2 b+1<2 a b-b c-b$. Factoring this gives $(2 b-1)(a-1)<b(2 a-c-1)$, or $\frac{a-1}{b}<\frac{2 a-c-1}{2 b-1}$ as desired.

We therefore concentrate on the second and third inequalities in (11). Subtracting $c$ from each expression in (11) and substituting $a=b c+r$ yields

$$
\frac{2 r-1}{2 b-1}<m-c \leq \frac{r}{b}=\frac{2 r}{2 b}
$$

Therefore, it will suffice to find a pair $b, r$ of positive integers satisfying (12).

Note that $m-c$ is the fractional part of $m$; since $m$ is not an integer, we have $m-c \in(0,1)_{\mathbb{R}}$. Therefore, it will suffice to show that $(0,1)$ is the union of intervals of the form $\left(\frac{2 r-1}{2 b-1}, \frac{2 r}{2 b}\right]$ for positive integers $b, r$. Indeed,

$$
\begin{aligned}
(0,1) & =\bigcup_{m \geq 0}\left(\frac{m}{m+1}, \frac{m+1}{m+2}\right] \\
& =\left(\frac{0}{1}, \frac{1}{2}\right] \cup\left(\frac{1}{2}, \frac{2}{3}\right] \cup\left(\frac{2}{3}, \frac{3}{4}\right] \cup \cdots
\end{aligned}
$$

and

$$
\begin{aligned}
\left(\frac{m}{m+1}, \frac{m+1}{m+2}\right] & =\bigcup_{s \geq 1}\left(\frac{2^{s} m+1}{2^{s}(m+1)+1}, \frac{2^{s} m+2}{2^{s}(m+1)+2}\right] \\
& =\left(\frac{2 m+1}{2 m+3}, \frac{2 m+2}{2 m+4}\right] \cup\left(\frac{4 m+1}{4 m+5}, \frac{4 m+2}{4 m+6}\right] \cup\left(\frac{8 m+1}{8 m+9}, \frac{8 m+2}{8 m+10}\right] \cup \cdots
\end{aligned}
$$


establishing (12), as desired.

3.2. Equivalent characterizations. One of the equivalent phrasings of $m$-Laman independence involves the Tutte polynomial $T_{M}(x, y)$ of $M$, a fundamental isomorphism invariant of the matroid $M$. For background on the Tutte polynomial, see the excellent survey article by Brylawski and Oxley [2].

Given a subset $A$ of the ground set $E$, denote by $\bar{A}$ the matroid closure or span of $A$. If $A=\bar{A}$, then $A$ is called a flat of $M$.

Proposition 3.3. Let $M$ be a matroid on ground set $E$ with rank function $r$, and fix $m \in(1, \infty)_{\mathbb{R}}$.

Then the following are equivalent:

(i) $E$ is $m$-Laman independent, that is, $\mathcal{L}^{m}(M)=2^{E}$ (the power set of $E$ ).

(ii) $m \cdot r(\bar{A})>|\bar{A}|$ for every nonempty subset $A \subseteq E$. (Equivalently, $m \cdot r(F)>|F|$ for every flat $F$ of M.)

(iii) The Tutte polynomial specialization $T_{M}\left(q^{m-1}, q\right)$ is monic of degree $(m-1) r(M)$.

Note that in (iii) we must allow (non-integral) real number exponents for a "polynomial" in $q$, but the notions of "degree" and "monic" for such polynomials should still be clear. The connection between the Tutte polynomial and rigidity of graphs was observed by the second author in $[8, \S 6]$.

Proof. The equivalence of (i) and (ii) is clear from the definition of $m$-Laman independence since $r(\bar{A})=r(A)$ and $|\bar{A}| \geq|A|$ for any $A \subseteq E$.

For the equivalence of (i) and (iii) we use Whitney's corank-nullity formula [2, eq. 6.13] for the Tutte polynomial:

$$
T_{M}(x, y)=\sum_{A \subseteq E}(x-1)^{r(M)-r(I)}(y-1)^{|I|-r(I)}
$$

Substituting $x=q^{m-1}$ and $y=q$ gives an expression for $T_{M}\left(q^{m-1}, q\right)$ as a sum of terms indexed by subsets $A \subseteq E$, each of which is a monic polynomial in $q$ of degree

$$
(m-1) r(M)-m \cdot r(A)+|A| \text {. }
$$

Thus $T_{M}\left(q^{m-1}, q\right)$ will have degree at most $(m-1) r(M)$ if and only if $m \cdot r(A) \geq|A|$ for all subsets $A \subseteq E$. Furthermore, since the term indexed by $A=\varnothing$ is monic of degree $(m-1) r(M)$, the whole polynomial $T_{M}\left(q^{m-1}, q\right)$ will be monic of degree $(m-1) r(M)$ if and only if $m \cdot r(A)>|A|$ for every nonempty subset $A$, that is, if and only if $E$ is $m$-Laman independent.

Suppose that $m=d$ is a positive integer, so that $\mathcal{L}^{d}(M)$ is a matroid complex. Here $d$-Laman independence has two more equivalent formulations, one of which extends a classical result in the rigidity theory of graphs.

Recski's Theorem [13]. Let $G=(V, E)$ be a graph, and let $E^{\prime}$ be a spanning set of edges of size $2|V|-3$. Then $E^{\prime}$ is a 2-rigidity basis if and only if for any $e \in E^{\prime}$, we can partition the multiset $E^{\prime} \cup\{e\}$ (that is, adding an extra copy of e to $E^{\prime}$ ) into two disjoint spanning trees of $G$.

This notion can be naturally extended to arbitrary matroids and dimensions.

Definition 3.4. Let $M$ be a matroid on $E$. We say that $E$ is $d$-Recski independent if for any element $e \in E$, the multiset $E \cup\{e\}$ can be partitioned into $d$ disjoint independent sets for $M$.

We wish to show that this purely matroidal condition is equivalent to the purely matroidal condition of $d$-Laman independence. To prove this, we use a powerful classic result of Edmonds.

Edmonds' Decomposition Theorem [3, Theorem 1]. Let $M$ be a matroid of rank $r$ on ground set E. Then $E$ has a decomposition $E=I_{1} \sqcup \cdots \sqcup I_{d}$ into disjoint independent sets $I_{j}$ for each $j=1, \ldots, d$ if and only if $d \cdot r(A) \geq|A|$ for every subset $A \subseteq E$.

Definition 3.5. Let $M$ be a matroid on $E$. A $d$-Edmonds decomposition of $M$ is a family of independent sets $I_{1}, \ldots, I_{d}$ whose disjoint union is $E$, with the following property: given subsets $I_{1}^{\prime} \subseteq I_{1}, \ldots, I_{d}^{\prime} \subseteq I_{d}$ with not all $I_{i}^{\prime}$ empty, then it is not the case that $\overline{I_{1}^{\prime}}=\overline{I_{2}^{\prime}}=\cdots=\overline{I_{d}^{\prime}}$. 
Theorem 3.6. Let $M$ be a matroid on ground set $E$, and let $d$ be a positive integer. Then the following are equivalent:

(i) E has a d-Edmonds decomposition;

(ii) $E$ is $d$-Laman independent;

(iii) $E$ is d-Recski independent.

Proof. (ii) $\Rightarrow$ (i): Suppose that $E$ is $d$-Laman independent. By Edmonds' Theorem, the set $E$ can be partitioned into disjoint independent sets $I_{1}, \ldots, I_{d}$. We claim that every such family is a $d$-Edmonds decomposition. Indeed, suppose that $I_{1}^{\prime} \subseteq I_{1}, \ldots, I_{d}^{\prime} \subseteq I_{d}$ all have the same span, with not all $I_{j}^{\prime}$ empty. Since the $I_{j}$ are independent, the $I_{j}^{\prime}$ all have the same cardinality, say $s$. Then $A:=I_{1}^{\prime} \sqcup \ldots \sqcup I_{d}^{\prime}$ is nonempty and has rank $s$ and cardinality $d s$, which violates the $d$-Laman independence of $E$.

(i) $\Rightarrow$ (ii): Let $I_{1}, \ldots, I_{d}$ be a $d$-Edmonds decomposition of $M$. Let $A \subseteq E$ be nonempty, and $A_{j}:=A \cap I_{j}$. Then

$$
|A|=\sum_{j=1}^{d}\left|A_{j}\right|=\sum_{j=1}^{d} r\left(A_{j}\right) \leq \sum_{i=1}^{d} r(A)=d \cdot r(A) .
$$

However, equality cannot hold: it would force $r\left(A_{j}\right)=r(A)$ for each $j$, so that each $A_{j}$ has the same span as $A$, violating the definition of a $d$-Edmonds decomposition. Hence $|A|<d \cdot r(A)$ as desired.

(ii) $\Rightarrow$ (iii): Suppose that $E$ is $d$-Laman independent. Consider the matroid $M^{\prime}$ given by cloning any $e \in E$ as in the definition of $d$-Recski independence, so that the ground set of $M^{\prime}$ is $E^{\prime}=E \cup\{e\}$. We claim that $\left|A^{\prime}\right| \leq d \cdot r\left(A^{\prime}\right)$ for each $A^{\prime} \subseteq E^{\prime}$. Indeed, either $A^{\prime} \subseteq E$, when $\left|A^{\prime}\right|<d \cdot r\left(A^{\prime}\right)$, or else $A^{\prime}=A \cup\{e\}$ with $A \subseteq E$, when $\left|A^{\prime}\right|=|A|+1<d \cdot r(A)+1$, so $\left|A^{\prime}\right| \leq d \cdot r(A) \leq d \cdot r\left(A^{\prime}\right)$. By Edmonds' Theorem, $E^{\prime}$ can be partitioned into $d$ disjoint independent subsets. It follows that $M$ is $d$-Recski independent.

(iii) $\Rightarrow$ (ii): Suppose that $E$ is not $d$-Laman independent, i.e., it has a subset $A$ with $|A| \geq d \cdot r(A)$. Let $a \in A$. The set $A \cup\{a\} \subseteq E \cup\{a\}$ has rank $r(A)$ and cardinality $|A|+1$, so $|A \cup\{a\}|>d \cdot r(|A \cup\{a\}|)$. By Edmonds' Theorem, $E \cup\{a\}$ cannot be partitioned into $d$ independent sets. Hence $E$ is not $d$-Recski independent.

3.3. Digression on polymatroids. As we have seen in Theorem 3.1 (ii), when $m$ is not an integer, the Laman complex $\mathcal{L}^{m}(M)$ need not form the collection of independent sets of a matroid. However, $\mathcal{L}^{m}(M)$ is related to a more general (and less well-known) object called a polymatroid, as we now explain. (The results of this section will not be necessary for the remainder of the paper.)

We review the definition of a polymatroid, using its characterizations by monotone submodular rank functions (see [17, Chapter 18]).

Definition 3.7. Fix the ground set $E=[n]$. A function $\rho: 2^{E} \rightarrow \mathbb{R}_{\geq 0}$ is the rank function of a polymatroid on $E$ if it is

- monotone: $\rho(A) \leq \rho(B)$ whenever $A \subseteq B \subseteq E$;

- submodular: $\rho(A \cup B)+\rho(A \cap B) \leq \rho(A)+\rho(B)$ for all $A, B \subseteq E$; and

- normalized: $\rho(\varnothing)=0$.

The polymatroid associated with $\rho$ is the convex polytope

$$
P_{\rho}:=\left\{x \in \mathbb{R}_{\geq 0}^{n}: \sum_{a \in A} x_{a} \leq \rho(A) \text { for all } A \subseteq E\right\},
$$

also called the set of independent vectors of the polymatroid.

Note that, for all $A \subseteq E$, the characteristic vector $\chi_{A} \in \mathbb{R}^{n}$ is independent for $\rho$ if and only if $\rho(A)=|A|$.

Our goal is to show the following:

Proposition 3.8. For every loopless matroid $M$ on ground set $E=[n]$, and every real number $m \in(1, \infty)_{\mathbb{R}}$, there is a polymatroid rank function $\rho$ on $E$ with the following property: $A \subseteq E$ is $m$-Laman independent if and only if its characteristic vector is independent for $\rho$.

The proof uses two standard lemmas. 
Lemma 3.9. [1, Lemma 6.15] If $f: 2^{E} \rightarrow \mathbb{R}_{\geq 0}$ is monotone, submodular, and normalized, then so is the function $r_{f}: 2^{E} \rightarrow \mathbb{R}_{\geq 0}$ defined by

$$
r_{f}(A):=\min _{A^{\prime} \subseteq A}\left\{f\left(A^{\prime}\right)+\left|A \backslash A^{\prime}\right|\right\} .
$$

Lemma 3.10. [19, Proposition A.3.1] For a monotone, submodular, normalized function $f: 2^{E} \rightarrow \mathbb{R}_{\geq 0}$ with associate function $r_{f}$ as above, the following are equivalent:

(a) $\left|A^{\prime}\right| \leq f\left(A^{\prime}\right)$ for all $A^{\prime} \subseteq A$.

(b) $\left|A^{\prime}\right| \leq r_{f}\left(A^{\prime}\right)$ for all $A^{\prime} \subseteq A$.

(c) $r_{f}(A)=|A|$.

Proof of Proposition 3.8. Let $\epsilon \in\left(0, \frac{1}{r(M)}\right)_{\mathbb{R}}$, and define $f: 2^{E} \rightarrow \mathbb{R}_{\geq 0}$ by

$$
f(A)=(m-\epsilon) r(A) .
$$

Note that $f$ is monotone, submodular, and normalized, because the rank function $r$ of any loopless matroid has these properties. By Lemma 3.9, the function $\rho:=r_{f}$ shares these properties, hence also defines a polymatroid rank function on $E$.

Since $M$ is loopless, for all $\mathcal{A} \neq \varnothing$, one has $\operatorname{mr}(A)>|A|$ if and only if $(m-\epsilon) r(A) \geq|A|$. Consequently

$$
\begin{aligned}
A \in \mathcal{L}^{m}(M) & \Longleftrightarrow f\left(A^{\prime}\right) \geq\left|A^{\prime}\right| \quad \text { for all nonempty } A^{\prime} \subseteq A \\
& \Longleftrightarrow f\left(A^{\prime}\right) \geq\left|A^{\prime}\right| \quad \text { for all } A^{\prime} \subseteq A \\
& \Longleftrightarrow \rho(A)=r_{f}(A)=|A| .
\end{aligned}
$$

Here the last equality uses Lemma 3.10 .

\section{Slope indePEndence AND the SPACE OF Photos}

In [7] and [9], the second author studied the picture space of a graph $G$, the algebraic variety of point-line arrangements in $d$-dimensional space with an incidence structure given by $G$. The rigidity-theoretic behavior of $G$ controls the geometry of the picture space to a great extent; for instance, the picture space is irreducible if and only if $G$ is $d$-parallel independent.

In this section, we study the space $X_{k, d}(M)$ of $(k, d)$-photos, which is well-defined for any matroid $M$ equipped with a representation. The photo space plays a role analogous to that of the picture space of a graph, ${ }^{1}$ and the techniques we use to study it are similar to those of [7]. In particular, $X_{k, d}(M)$ provides a geometric interpretation of $m$-Laman independence for all rational numbers $m>1$ : it is equivalent to the space of $(k, d)$-photos.

Throughout this section, we work with a matroid $M$ represented over a field $\mathbb{F}$ by nonzero ${ }^{2}$ vectors $v_{1}, \ldots, v_{n} \in \mathbb{F}^{r}$. In addition, let $0<k<d$ be integers, and write $m=\frac{d}{d-k}$. Recall (Definition 2.2) that the space of $(k, d)$-photos of $M$ is

$$
\left\{(\varphi, W) \in \operatorname{Hom}_{\mathbb{F}}\left(\mathbb{F}^{r}, \mathbb{F}^{d}\right) \times \mathbb{G} r\left(k, \mathbb{F}^{d}\right)^{n}: \varphi\left(v_{i}\right) \in W_{i} \text { for all } 1 \leq i \leq n\right\} .
$$

Note that the photo space is an algebraic subset of $\operatorname{Hom}_{\mathbb{F}}\left(\mathbb{F}^{r}, \mathbb{F}^{d}\right) \times \mathbb{G} r\left(k, \mathbb{F}^{d}\right)^{n}$, hence a scheme over $\mathbb{F}$. The symbol $X_{k, d}(M)$ is a slight abuse of notation; as defined, the photo space depends on the representation $\left\{v_{i}\right\}$, and it is not at all clear to what extent it depends only on the structure of $M$ as an abstract matroid. (We will return to this natural question later.)

A key tool in our analysis is a disjoint decomposition of the photo space into irreducible algebraic subsets called cellules (in analogy to [7]). For each photo $(\varphi, W), \operatorname{ker} \varphi$ is a linear subspace of $\mathbb{F}^{r}$, hence intersects $E$ in some flat $F$ of $M$. The idea is to classify photos according to what this flat is.

Definition 4.1. For each flat $F \subseteq E$, the corresponding cellule is

$$
X_{k, d}^{F}(M)=\left\{(\varphi, W) \in X_{k, d}(M): \operatorname{ker} \varphi \cap E=F\right\} .
$$

\footnotetext{
${ }^{1}$ The reader should be warned not to take this analogy too literally: the picture space of a graph is not an instance of the photo space of a matroid!

${ }^{2}$ Our assumption that $M$ contains no loops is purely for convenience; trivial (but slightly annoying) modifications are necessary when loops are present.
} 
By definition, each photo belongs to exactly one cellule; that is, $X_{k, d}(M)$ decomposes as a disjoint union of the cellules. Of particular importance are the two extreme cases:

I. The cellule $X_{k, d}^{\varnothing}(M)$ corresponding to the empty flat $\varnothing$ is called the non-annihilating cellule. It is a Zariski open subset of $X_{k, d}(M)$, defined by the conjunction of open conditions

$$
\varphi\left(v_{i}\right) \neq 0, \quad \forall i=1, \ldots, n .
$$

II. The cellule $X_{k, d}^{E}(M)$ corresponding to the improper flat $E$ is called the degenerate cellule. It is precisely $\{0\} \times \mathbb{G} r\left(k, \mathbb{F}^{d}\right)^{n}$, where 0 is the zero map $\mathbb{F}^{r} \rightarrow \mathbb{F}^{d}$.

Proposition 4.2. Let $M$ and $X_{k, d}(M)$ be as above.

(i) The natural projection map

$$
X_{k, d}^{\varnothing}(M) \rightarrow \operatorname{Hom}_{\mathbb{F}}\left(\mathbb{F}^{r}, \mathbb{F}^{d}\right)
$$

gives $X_{k, d}^{\varnothing}(M)$ the structure of an algebraic fiber bundle, with fiber $\mathbb{G} r\left(k-1, \mathbb{F}^{d-1}\right)$ and base the Zariski open subset of $\operatorname{Hom}_{\mathbb{F}}\left(\mathbb{F}^{r}, \mathbb{F}^{d}\right)$ defined by (13). In particular, $\operatorname{dim} X_{k, d}^{\varnothing}(M)=d r+n(k-1)(d-k)$.

(ii) For each flat $F, X_{k, d}^{F}(M) \cong X_{k, d}^{\varnothing}(M / F) \times \mathbb{G} r\left(k, \mathbb{F}^{d}\right)^{F}$. Consequently, $X_{k, d}^{F}(M)$ is an irreducible subvariety of $X_{k, d}(M)$, with dimension given by the formula

$$
\operatorname{dim} X_{k, d}^{F}(M)=d(r-r(F))+(n-|F|)(k-1)(d-k)+|F| k(d-k) .
$$

The preceding assertions are more or less immediate from the definition of cellules and the standard fact that the Grassmannian $\mathbb{G} r\left(k, \mathbb{F}^{d}\right)$ has dimension $k(d-k)$.

As in (7), let $\pi$ denote the projection map

$$
\operatorname{Hom}_{\mathbb{F}}\left(\mathbb{F}^{r}, \mathbb{F}^{d}\right) \times \mathbb{G} r\left(k, \mathbb{F}^{d}\right)^{n} \stackrel{\pi}{\longrightarrow} \mathbb{G} r\left(k, \mathbb{F}^{d}\right)^{n},
$$

and define $M$ to be $(k, d)$-slope independent if $\pi X_{k, d}^{\varnothing}(M)$ is Zariski dense in $\mathbb{G} r\left(k, \mathbb{F}^{d}\right)^{n}$. We will denote the Zariski closure of a set $Z$ by $\bar{Z}$.

Theorem 4.3. Let $M$ be a matroid with rank function $r$, represented by vectors $v_{1}, \ldots, v_{n} \in \mathbb{F}^{r}$. Fix positive integers $0<k<d$, and let $m=\frac{d}{d-k}$.

Then the following are equivalent:

(i) $M$ is $(k, d)$-slope independent, i.e., $\pi X_{k, d}^{\varnothing}(M)$ is dense in $\mathbb{G} r\left(k, \mathbb{F}^{d}\right)^{n}$.

(ii) $M$ is $m$-Laman independent, i.e., $m \cdot r(F)>|F|$ for every nonempty flat $F$ of $M$.

(iii) $\operatorname{dim} X_{k, d}^{F}(M)<\operatorname{dim} X_{k, d}^{\varnothing}(M)$ for every nonempty flat $F$ of $M$.

(iv) The photo space $X_{k, d}(M)$ is irreducible.

(v) The photo space $X_{k, d}(M)$ coincides with the Zariski closure $\overline{X_{k, d}^{\varnothing}(M)}$ of its non-annihilating cellule.

Proof. (ii) $\Leftrightarrow$ (iii): Compare the cellule dimension formula (14) dimension with the definition of $m$-Laman independence (Definition 2.1).

(i) $\Rightarrow$ (ii): For a nonempty flat $F$, write $\left.M\right|_{F}$ for the restriction of $M$ to $F$. Consider the commutative diagram

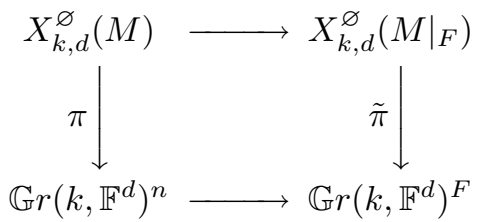

in which the top horizontal morphism restricts the photo map $\varphi$ to the linear span $\mathbb{F}(F)$ of the vectors in $F$, while forgetting the $k$-planes $\left\{W_{i}\right\}_{i \in E \backslash F}$. Both vertical arrows are projections as in (7); we use the tilde on the right-hand map to distinguish them in what follows. Note that when $\varphi$ is non-annihilating, its restriction to the span of $F$ will also be non-annihilating. Moreover, the bottom horizontal morphism is surjective. 
Now assume that condition (i) holds. Since the image of $\pi$ is Zariski dense in the target, so is the image of $\tilde{\pi}$. Therefore

$$
d \cdot r(F)+|F|(k-1)(d-k)=\operatorname{dim} X_{k, d}^{\varnothing}\left(M_{F}\right) \geq \operatorname{dim} \mathbb{G} r\left(k, \mathbb{F}^{d}\right)^{F}=|F| k(d-k),
$$

or in other words, $d \cdot r(F) \geq(d-k)|F|$. However, scaling a non-annihilating map $\varphi$ by an element of $\mathbb{F}^{\times}$ does not change the line spanned by any $\varphi\left(v_{i}\right)$. Therefore every fiber of $\tilde{\pi}$ is at least one-dimensional. Put differently, when restricted to $X_{k, d}^{\varnothing}\left(\left.M\right|_{F}\right)$, the morphism $\tilde{\pi}$ factors through a $(d \cdot r(F)-1)+|F|(k-1)(d-k)$ dimensional space of projectivized non-annihilating maps $\varphi$ in $\mathbb{P}\left(\operatorname{Hom}_{\mathbb{F}}\left(\mathbb{F}(F), \mathbb{F}^{d}\right)\right.$.

Hence, for every nonempty flat $F$, we have the strict inequality $d \cdot r(F)>(d-k)|F|$, or equivalently $m \cdot r(F)>|F|$, which is (ii).

(iv) $\Leftrightarrow(\mathrm{v})$ : Since $X_{k, d}^{\varnothing}(M)$ is Zariski open in $X_{k, d}(M)$, its closure $\overline{X_{k, d}^{\varnothing}(M)}$ is one of the irreducible components of $X_{k, d}(M)$. Thus the full photo space is irreducible if and only if the non-annihilating photos are dense.

$(v) \Rightarrow(\mathrm{i})$ : Suppose that (v) holds. Then (i) follows from the observation that

$$
\overline{\pi\left(X_{k, d}^{\varnothing}(M)\right)} \supset \pi\left(\overline{X_{k, d}^{\varnothing}(M)}\right) \supset \pi\left(X_{k, d}^{E}(M)\right)=\left(\mathbb{P}_{\mathbb{F}}^{d-1}\right)^{E},
$$

(the first inclusion is standard, and the second is implied by $(\mathrm{v})$ ).

(iii) $\Rightarrow$ (iv): We begin by finding an upper bound for the codimension of every component of the photo space. Note that $X_{k, d}(M)=\bigcap_{i=1}^{n} Z_{i}$, where

$$
Z_{i}=\left\{(\varphi, W) \in \operatorname{Hom}\left(\mathbb{F}^{r}, \mathbb{F}^{d}\right) \times \mathbb{G} r\left(k, \mathbb{F}^{d}\right)^{n}: \varphi\left(v_{i}\right) \in W_{i}\right\}
$$

Let

$$
\begin{aligned}
Z_{i}^{\prime} & =\left\{(\varphi, W) \in Z_{i}: \varphi\left(v_{i}\right) \neq 0\right\} \\
Z_{i}^{\prime \prime} & =\left\{(\varphi, W) \in Z_{i}: \varphi\left(v_{i}\right)=0\right\} .
\end{aligned}
$$

Note that $Z_{i}^{\prime}$ has codimension $d-k$ in $\operatorname{Hom}_{\mathbb{F}}\left(\mathbb{F}^{r}, \mathbb{F}^{d}\right) \times \mathbb{G} r\left(k, \mathbb{F}^{d}\right)^{n}$. Additionally, $Z_{i}^{\prime \prime}$ is contained in the Zariski closure of $Z_{i}^{\prime}$, because the condition $\varphi\left(v_{i}\right) \in W_{i}$ (expressed using the Plücker coordinates of $W_{i}$ ) is satisfied also when $\varphi\left(v_{i}\right)=0$. Therefore, every $Z_{i}$ has codimension $d-k$, and every irreducible component of their intersection $X_{k, d}(M)$ has codimension at most $n(d-k)$. On the other hand, by the cellule dimension formula (14), $n(d-k)$ is precisely the codimension of the non-annihilating cellule $X_{k, d}^{\varnothing}(M)$. Hence every irreducible component of $X_{k, d}(M)$ has dimension at least as large as that of $X_{k, d}^{\varnothing}(M)$.

Now suppose that (iii) holds, so that $\operatorname{dim} X_{k, d}^{F}(M)<\operatorname{dim} X_{k, d}^{\varnothing}(M)$ for every $F \neq \varnothing$. Since the cellules are all irreducible and disjointly decompose $X_{k, d}(M)$, the irreducible components of $X_{k, d}(M)$ must be exactly the closed cellules $\overline{X_{k, d}^{F}(M)}$ that are contained in the closure of no other cellule. On the other hand, by the previous paragraph, every such cellule must have its dimension at least that of $\operatorname{dim} X_{k, d}^{\varnothing}(M)$, and by (iii) the only possibility is $F=\varnothing$. Therefore $\overline{X_{k, d}^{\varnothing}(M)}$ is the unique irreducible component.

The equivalence of (i) and (ii) in Theorem 4.3 immediately gives the following equality between the slope and Laman complexes.

Corollary 4.4. Let $m \in \mathbb{Q} \cap(1, \infty)_{\mathbb{R}}$. Write $m$ as $\frac{d}{d-k}$, where $0<k<d$ are integers.

$$
\text { Then } \mathcal{S}^{k, d}(M)=\mathcal{L}^{m}(M) \text {. }
$$

Remark 4.5. The condition $d \geq 2$ is implicit in Corollary 4.4. However, there is a sense in which the result is still valid for $d=1$. Take $k=1$, so that the result asserts that

$$
\mathcal{S}^{1, d}(M)=\mathcal{L}^{\frac{d}{d-1}}(M)
$$

Now, if one establishes conventions properly, this equality remains valid as $d$ approaches 1 , so that $m=\frac{d}{d-1}$ approaches infinity. That is,

$$
\mathcal{S}^{1,1}(M)=\mathcal{L}^{\infty}(M)=2^{E}
$$


Indeed, the full simplex $2^{E}$ is logically equal to $\mathcal{S}^{1,1}(M)$ : there is only one possible line through any point in $\mathbb{F}^{1}$, so the projection map $\pi$ is dense. Meanwhile, it is easy to see that $\mathcal{L}^{\infty}(M)=2^{E}$, where we have defined

$$
\mathcal{L}^{\infty}(M):=\lim _{m \rightarrow \infty} \mathcal{L}^{m}(M)
$$

Remark 4.6. For a given matroid $M$ and irrational number $m$, it is not hard to see that there exists a rational number $\tilde{m}$, chosen sufficiently close to $m$, such that $\mathcal{L}^{\tilde{m}}(M)=\mathcal{L}^{m}(M)$. Therefore, Corollary 4.4 actually gives a geometric interpretation for every instance of Laman independence.

Remark 4.7. Another surprising consequence of Corollary 4.4 is that $(k, d)$-slope-independence is invariant under simultaneously scaling $k$ and $d$. That is, if $\lambda>0$ is an integer, then the Corollary implies that

$$
\mathcal{S}^{k, d}(M)=\mathcal{S}^{\lambda k, \lambda d}(M)
$$

Moreover, if $d$ is divisible by $k$, then $m=d /(d-k)$ is an integer and $\mathcal{S}^{k, d}(M)=\mathcal{L}^{m}(M)$ is in fact a matroid by Theorem 3.1 (i). The geometry behind these phenomena is far from clear.

A natural question is to determine the singularities of the photo space. While we cannot do this in general, we can at least say exactly for which matroids $X_{k, d}(M)$ is smooth. The result and its proof are akin to [9, Proposition 15], and do not depend on the parameters $k$ and $d$.

Proposition 4.8. Let $M$ be a loopless matroid equipped with a representation $\left\{v_{1}, \ldots, v_{n}\right\}$ as above. Then, for all integers $0<k<d$, the photo space $X=X_{k, d}(M)$ is smooth if and only if $M$ is Boolean (that is, every ground set element is an isthmus).

The assumption of looplessness is harmless, because if $v_{i}$ is a loop, then $X_{k, d}(M) \cong \mathbb{G} r\left(k, \mathbb{F}^{d}\right) \times X_{k, d}(M \backslash v)$, so $X_{k, d}(M)$ is smooth if and only if $X_{k, d}(M \backslash v)$ is.

Proof. First, note that the photo space of a direct sum of matroids is precisely the product of their photo spaces (this can be seen by writing the matrix for a picture of the direct sum in block-diagonal form). In particular, if $M$ is Boolean, then

$$
X \cong \prod_{i=1}^{n}\left\{\left(\varphi_{i}, W_{i}\right) \in \mathbb{F}^{d} \times \mathbb{G} r\left(k, \mathbb{F}^{d}\right): \varphi_{i}\left(v_{i}\right) \in W_{i}\right\}
$$

and each factor in the product is a copy of the total space of the tautological $k$-plane bundle over $\mathbb{G} r\left(k, \mathbb{F}^{d}\right)$. In particular, $X$ is smooth.

Now suppose that $M$ is not Boolean; in particular $n>r$. Recall from Proposition 4.2 that the nonannihilating cellule has dimension $d r+n(k-1)(d-k)$. Near each non-annihilating photo $\Omega$, the photo space looks locally like an affine space of this dimension; in particular, the tangent space $T_{\Omega}(X)$ has dimension

$$
\operatorname{dim} T_{\Omega}(X)=d r+n(k-1)(d-k)
$$

Let $\Phi=(\varphi, W)$ be a "very degenerate" photo; that is, $\varphi=0$ and all the $k$-planes $W_{i}$ coincide. Each $W_{i}$ can be moved freely throughout the $i$ th Grassmannian, giving $n \cdot \operatorname{dim} \mathbb{G} r\left(k, \mathbb{F}^{d}\right)=n k(d-k)$ independent tangent vectors to $X$ at $\Phi$. On the other hand, we can also vary the map $\varphi$ throughout $\operatorname{Hom}\left(\mathbb{F}^{r}, W_{i}\right)$, giving $k r$ more tangent directions that are linearly independent of those just mentioned. Therefore

$$
\operatorname{dim} T_{\Phi}(X) \geq n k(d-k)+k r .
$$

Comparing (17) and (18), and doing a little algebra, we find that

$$
\operatorname{dim} T_{\Phi}(X)-\operatorname{dim} T_{\Omega}(X) \geq(d-k)(n-r)>0 .
$$

That is, not all points of $X$ have the same tangent space dimension. Therefore $X$ cannot be smooth. 


\section{Counting photos}

Although it will not be needed in the sequel, we digress to prove an enumerative result, possibly of independent interest, about the photo space: when working over a finite field, the cardinality $\left|X_{k, d}(M)\right|$ is an evaluation of the Tutte polynomial $T_{M}(x, y)$.

We refer the reader to [2] for details on the Tutte polynomial. In what follows, we write $M \backslash v$ and $M / v$ respectively for the deletion and contraction of $M$ with respect to an element $v$ of its ground set. We also dispense with the assumption from the previous section that $M$ contains no loops. On the other hand, we add the assumption that the representing vectors $v_{1}, \ldots, v_{n} \in \mathbb{F}^{r}$ actually span $\mathbb{F}^{r}$; in other words, $r(M)=r$. This represents no loss of generality; it is easy to check that when $r(M)<r$, there is a natural isomorphism

$$
X_{k, d}(M) \cong \operatorname{Hom}_{\mathbb{F}}\left(\mathbb{F}^{r-r(M)}, \mathbb{F}^{d}\right) \times X_{k, d}\left(M^{\prime}\right),
$$

where $M^{\prime}$ is represented by the same vectors $v_{1}, \ldots, v_{r}$, regarded as elements of the $r(M)$-dimensional subspace of $\mathbb{F}^{r}$ that they span.

The following fact [2, Corollary 6.2.6] is a standard tool for converting deletion-contraction recurrences to Tutte polynomial evaluations. We need the dual matroid $M^{\perp}$, characterized as follows: when $M$ is represented by the columns $v_{1}, \ldots, v_{n}$ of an $r \times n$ matrix of rank $r$ as above, the dual $M^{\perp}$ is represented by the columns $v_{1}^{*}, \ldots, v_{n}^{*}$ of an $(n-r) \times n$ matrix of rank $n-r$, with the property that the row space of $M^{\perp}$ is the nullspace of $M$, and vice versa. (In purely combinatorial terms, the bases of $M^{\perp}$ are the complements of bases of $M$.)

Proposition 5.1. Let $\Psi(M)$ be an invariant of matroids taking values in a commutative ring $R$, with the following properties:

(T1) For all matroids $M_{1}, M_{2}, \Psi\left(M_{1} \oplus M_{2}\right)=\Psi\left(M_{1}\right) \Psi\left(M_{2}\right)$.

(T2) When the ground set of $M$ consists of a single isthmus, $\Psi(M)=c$.

(T3) When the ground set of $M$ consists of a single loop, $\Psi(M)=d$.

(T4) When $v$ is neither a loop nor an isthmus of $M, \Psi(M)=a \Psi(M \backslash v)+b \Psi(M / v f)$. Then

$$
\Psi(M)=a^{r\left(M^{\perp}\right)} b^{r(M)} T_{M}\left(\frac{c}{b}, \frac{d}{a}\right) .
$$

Recall [14, Proposition 1.3.18] that when $\mathbb{F}$ is a finite field with $q$ elements, the cardinality of the Grassmannian $\mathbb{G} r\left(k, \mathbb{F}^{d}\right)$ is given by the $q$-binomial coefficient

$$
\left[\begin{array}{l}
d \\
k
\end{array}\right]_{q}:=\frac{[d] !_{q}}{[k] !_{q}[d-k] !_{q}}
$$

where

$$
[n] !_{q}:=[n]_{q}[n-1]_{q} \cdots[2]_{q}[1]_{q}
$$

and

$$
[n]_{q}:=\frac{1-q^{n}}{1-q}=1+q+q^{2}+\cdots+q^{n-1} .
$$

We can now state the main result on counting photos.

Theorem 5.2. Let $\mathbb{F}$ be the finite field with $q$ elements. Let $M$ be a matroid of rank $r$, represented over $\mathbb{F}$ by vectors $v_{1}, \ldots, v_{n}$ spanning $\mathbb{F}^{r}$, and let $d \geq 2$. Then the number of $(k, d)$-photos of $M$ is

$$
\left|X_{k, d}(M)\right|=\left[\begin{array}{c}
d-1 \\
k-1
\end{array}\right]_{q}^{r\left(M^{\perp}\right)}\left(q^{k}\left[\begin{array}{c}
d-1 \\
k
\end{array}\right]_{q}\right)^{r(M)} T_{M}\left(\frac{[d]_{q}}{[d-k]_{q}}, \frac{[d]]_{q}}{[k]_{q}}\right)
$$

Proof. Abbreviate $X_{k, d}(M)$ by $X(M)$, and define $\Psi(M):=|X(M)|$. We must show that $\Psi$ satisfies the conditions of Proposition 5.1 with

$$
a=\left[\begin{array}{l}
d-1 \\
k-1
\end{array}\right]_{q}, \quad b=q^{k}\left[\begin{array}{c}
d-1 \\
k
\end{array}\right]_{q}, \quad c=q^{k}\left[\begin{array}{l}
d \\
k
\end{array}\right]_{q}, \quad d=\left[\begin{array}{l}
d \\
k
\end{array}\right]_{q} .
$$

(By an easy calculation, the arguments to the Tutte polynomial in the statement of the theorem are precisely $c / b$ and $d / a$.) 
Condition (T1) is straightforward. For (T2), if the ground set of $M$ consists of a single loop, then $X(M) \cong \mathbb{G} r\left(k, \mathbb{F}^{d}\right)$ has cardinality $\left[\begin{array}{l}d \\ k\end{array}\right]_{q}$.

If the ground set of $M$ consists of a single isthmus $v$, then a $(k, d)$-photo of $M$ is just a pair $(\varphi, W)$ where $\varphi: \mathbb{F}^{1} \rightarrow \mathbb{F}^{d}$ and $W$ is a $k$-plane containing $\varphi(v)$. Since the image vector $w:=\varphi(v)$ completely determines the map $\varphi$, a photo is equivalent to a pair $(w, W) \in \mathbb{F}^{d} \times \mathbb{G} r\left(k, \mathbb{F}^{d}\right)$ satisfying $w \in W$. Thus the space $X_{k, d}(M)$ is isomorphic to the tautological $k$-plane bundle over $\mathbb{G} r\left(k, \mathbb{F}^{d}\right)$, and its cardinality is $q^{k}\left[\begin{array}{l}d \\ k\end{array}\right]_{q}$, establishing condition (T3).

The verification of (T4) is the crux of the proof. If $v$ is neither a loop nor an isthmus of $M$, we have the following commutative diagram:

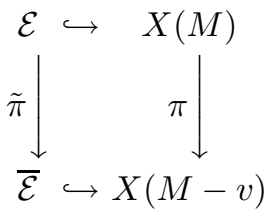

The map $\pi$ sends a $(k, d)$-photo of $M$ to a photo of $M \backslash v$ by forgetting the $k$-plane corresponding to the vector $v$. The map $\tilde{\pi}$ is the restriction of $\pi$ to the source and target

$$
\begin{array}{llll}
\mathcal{E} & :=\{(\varphi, W) \in X(M): & \varphi(v)=0\} & \cong X(M / e) \times \mathbb{G} r\left(k, \mathbb{F}^{d}\right) \\
\overline{\mathcal{E}}:=\{(\varphi, W) \in X(M \backslash v): & \varphi(v)=0\} & \cong X(M / e)
\end{array}
$$

and corresponds to the projection of $X(M / e) \times \mathbb{G r}\left(k, \mathbb{F}^{d}\right)$ onto its first factor. Meanwhile, the restriction

$$
X(M) \backslash \mathcal{E} \stackrel{\pi}{\longrightarrow} X(M \backslash v) \backslash \overline{\mathcal{E}}
$$

makes $X(M) \backslash \mathcal{E}$ into a bundle with fiber $\mathbb{G} r\left(k-1, \mathbb{F}^{d-1}\right)$. Consequently

$$
|X(M) \backslash \mathcal{E}|=\left[\begin{array}{l}
d-1 \\
k-1
\end{array}\right]_{q}|X(M \backslash v) \backslash \overline{\mathcal{E}}|
$$

and

$$
\begin{aligned}
\Psi(M) & =|\mathcal{E}|+\left[\begin{array}{l}
k-1 \\
d-1
\end{array}\right]_{q}(\Psi(M \backslash v)-|\overline{\mathcal{E}}|) \\
& =\left[\begin{array}{l}
d-1 \\
k-1
\end{array}\right]_{q} \Psi(M \backslash v)+\left[\begin{array}{l}
d \\
k
\end{array}\right]_{q} \Psi(M / v)-\left[\begin{array}{l}
d-1 \\
k-1
\end{array}\right]_{q} \Psi(M / v) \\
& =\left[\begin{array}{l}
d-1 \\
k-1
\end{array}\right]_{q} \Psi(M \backslash v)+q^{k}\left[\begin{array}{c}
d-1 \\
k
\end{array}\right]_{q} \Psi(M / v)
\end{aligned}
$$

where the last equality uses the $q$-Pascal recurrence [14, Chapter $1, \S 1.3$, Equation (17b)]

$$
\left[\begin{array}{l}
d \\
k
\end{array}\right]_{q}=q^{k}\left[\begin{array}{c}
d-1 \\
k
\end{array}\right]_{q}+\left[\begin{array}{l}
d-1 \\
k-1
\end{array}\right]_{q} .
$$

Since the Tutte polynomial of $M$ does not depend on the choice of representation, neither does the number of photos. Theorem 5.2 also implies a curious symmetry between the number of photos of a matroid $M$ and of its dual $M^{\perp}$. Since $T_{M^{\perp}}(x, y)=T_{M}(y, x)\left[2\right.$, Prop. 6.2.4] and $\left[\begin{array}{l}d \\ k\end{array}\right]_{q}=\left[\begin{array}{c}d \\ d-k\end{array}\right]_{q}$, we have:

Corollary 5.3. Let $M$ and $M^{\perp}$ be dual represented matroids. Then

$$
q^{d \cdot r(M)}\left|X_{d-k, d}\left(M^{\perp}\right)\right|=q^{(d-k) n}\left|X_{k, d}(M)\right| .
$$

It would be nice to have a more direct explanation for Corollary 5.3. 
Remark 5.4. A topological commutative diagram analogous to (19) was exploited by the second author in [8] to compute the Poincaré series of picture spaces of graphs over $\mathbb{C}$ as an analogous Tutte polynomial evaluation. In contrast, when $\mathbb{F}=\mathbb{R}$ or $\mathbb{C}$, the topology of the photo space is much simpler. Indeed, there is a deformation retraction of $X_{k, d}(M)$ onto its degenerate cellule:

$$
\begin{aligned}
F:[0,1] \times X_{k, d}(M) & \rightarrow X_{k, d}^{E}(M) \\
(\lambda,(\varphi, W)) & \mapsto(\lambda \varphi, W) .
\end{aligned}
$$

Hence $X_{k, d}(M)$ is homotopy equivalent to the degenerate cellule $X_{k, d}^{\varnothing}(M)$, which is homeomorphic to $\mathbb{G} r\left(k, \mathbb{F}^{d}\right)^{n}$ (see Definition 4.1).

\section{Rigidity AND PARALLEL INDEPENDENCE}

In this section, we examine more closely the special cases $k=1$ and $k=d-1$ of $(k, d)$-slope independence for a represented matroid $M$. It turns out that they are intimately related to the $d$-dimensional generic rigidity matroid $\mathcal{R}^{d}(M)$ and the $d$-dimensional generic hyperplane-marking matroid $\mathcal{H}^{d}(M)$. Throughout the section, let $M$ be a matroid represented by vectors $E=\left\{v_{1}, \ldots, v_{n}\right\}$ spanning $\mathbb{F}^{r}$, and let $d>0$ be an integer.

6.1. Interpreting $\mathcal{R}^{d}(M)$ and $\mathcal{H}^{d}(M)$. Recall (Definition 2.3) that the $d$-dimensional rigidity matroid is represented over $\mathbb{F}(\varphi)$ by the vectors

$$
\left\{v_{i} \otimes \varphi\left(v_{i}\right)\right\}_{i=1}^{n} \subset \mathbb{F}^{r} \otimes_{\mathbb{F}} \mathbb{F}(\varphi)^{d} .
$$

where $\mathbb{F}(\varphi)$ is the extension of $\mathbb{F}$ by $d r$ transcendentals (the entries of the matrix $\varphi: \mathbb{F}^{r} \rightarrow \mathbb{F}(\varphi)^{d}$ ). The complex $\mathcal{R}^{d}(M)$ is defined to be the complex of independent sets of this matroid. The d-rigidity matrix $R^{d}(M)$ is the $n \times d r$ matrix whose rows represent $\mathcal{R}^{d}(M)$.

Recall also (Definition 2.4) that the $d$-dimensional hyperplane-marking matroid is represented over $\mathbb{F}(\varphi, n)$ by the vectors

$$
\left.\left\{v_{i} \otimes \eta_{i}\right)\right\}_{i=1}^{n} \subset \mathbb{F}^{r} \otimes_{\mathbb{F}} \mathbb{F}(\varphi, \eta)^{d} .
$$

where $\mathbb{F}(\varphi)$ is the extension of $\mathbb{F}$ by $d r+(d-1) n$ transcendentals (the $d r$ entries of the matrix $\varphi$, and the $(d-1) n$ coordinates of the normal vectors $\eta_{i}$ to $\left.\varphi\left(v_{i}\right)\right)$. The complex $\mathcal{H}^{d}(M)$ is defined to be the complex of independent sets of this matroid. Denote by $H^{d}(M)$ the $n \times d r$ matrix whose rows represent $\mathcal{H}^{d}(M)$.

To interpret $R^{d}(M)$ and $H^{d}(M)$, we study their (right) nullspaces. Both matrices have row vectors in $\mathbb{F}^{r} \otimes_{\mathbb{F}} \mathbb{F}^{d}$, so their nullvectors live in the same space. It will be convenient to freely use the identifications

$$
\mathbb{F}^{r} \otimes_{\mathbb{F}} \mathbb{F}^{d} \cong\left(\mathbb{F}^{r}\right)^{*} \otimes_{\mathbb{F}} \mathbb{F}^{d} \cong \operatorname{Hom}_{\mathbb{F}}\left(\mathbb{F}^{r}, \mathbb{F}^{d}\right) .
$$

The second of these isomorphisms is canonical; the first comes from identifying $\mathbb{F}^{r}$ and $\left(\mathbb{F}^{r}\right)^{*}$ by the standard bilinear form on $\mathbb{F}^{r}$,

$$
\langle x, y\rangle=\sum_{i=1}^{r} x_{i} y_{i},
$$

whose associated quadratic form is

$$
Q(x)=\langle x, x\rangle=\sum_{i=1}^{r} x_{i}^{2} .
$$

With these identifications, for every $\psi \in \mathbb{F}^{r} \otimes_{\mathbb{F}} \mathbb{F}^{d} \cong \operatorname{Hom}_{\mathbb{F}}\left(\mathbb{F}^{r}, \mathbb{F}^{d}\right), v \in \mathbb{F}^{r}$, and $x \in \mathbb{F}^{d}$, the induced bilinear form on $\mathbb{F}^{r} \otimes_{\mathbb{F}} \mathbb{F}^{d}$ has the property

$$
\langle v \otimes x, \psi\rangle=\langle x, \psi(v)\rangle .
$$

Proposition 6.1. Let $M$ be a matroid represented by $E$ as above, and let $\psi \in \mathbb{F}^{r} \otimes_{\mathbb{F}} \mathbb{F}^{d} \cong \operatorname{Hom}_{\mathbb{F}}\left(\mathbb{F}^{r}, \mathbb{F}^{d}\right)$.

(i) The vector $\psi$ lies in $\operatorname{ker} H^{d}(M)$ if and only if $(\varphi+\psi)\left(v_{i}\right)$ is normal to $\eta_{i}$ for all $i=1,2, \ldots, n$.

(In other words, the nullspace of $H^{d}(M)$ is the space of directions in which one can modify the map $\varphi$ while keeping the image of $v_{i}$ lying on the same hyperplane normal to $\eta_{i}$ for each $i$.) 
(ii) Provided that $\mathbb{F}$ does not have characteristic 2 , the vector $\psi$ lies in $\operatorname{ker} R^{d}(M)$ if and only if

$$
Q\left((\varphi+\epsilon \psi)\left(v_{i}\right)\right) \equiv Q\left(\varphi\left(v_{i}\right)\right) \bmod \epsilon^{2}
$$

for each $i=1,2, \ldots, n$.

(In other words, the nullspace of $R^{d}(M)$ is the space of infinitesimal modifications one can make to $\varphi$ while keeping the values of the quadratic form $Q$ on the images of the $v_{i}$ constant (up to first order) for each $i$.)

Proof. For (i), note that

$$
\begin{aligned}
\left\langle\eta_{i},(\varphi+\psi)\left(v_{i}\right)\right\rangle=0 & \Longleftrightarrow\left\langle\eta_{i}, \varphi\left(v_{i}\right)\right\rangle+\left\langle\eta_{i}, \psi\left(v_{i}\right)\right\rangle=0 \\
& \Longleftrightarrow\left\langle\eta_{i}, \psi\left(v_{i}\right)\right\rangle=0 \\
& \Longleftrightarrow\left\langle v_{i} \otimes \eta_{i}, \psi\right\rangle=0 .
\end{aligned}
$$

For (ii), the expression

$$
Q\left((\varphi+\epsilon \psi)\left(v_{i}\right)\right)=Q\left(\left(\varphi\left(v_{i}\right)\right)+2 \epsilon\left\langle\varphi\left(v_{i}\right), \psi\left(v_{i}\right)\right\rangle+\epsilon^{2} Q\left(\psi\left(v_{i}\right)\right)\right.
$$

is congruent to $Q\left(\varphi\left(v_{i}\right)\right)$ modulo $\epsilon^{2}$ if and only if $\left\langle\varphi\left(v_{i}\right), \psi\left(v_{i}\right)\right\rangle=0$ (since $\mathbb{F}$ does not have characteristic 2). But $\left\langle\varphi\left(v_{i}\right), \psi\left(v_{i}\right)\right\rangle=\left\langle v_{i} \otimes \varphi\left(v_{i}\right), \psi\right\rangle$, completing the proof.

Remark 6.2. Part (i) of Proposition 6.1 is a rephrasing of the following familiar fact from rigidity theory: the rigidity matrix $R^{d}(M)$ may be regarded as the Jacobian matrix (after scaling by $\frac{1}{2}$ ) of the map

$$
\begin{array}{lll}
\operatorname{Hom}_{\mathbb{F}}\left(\mathbb{F}^{r}, \mathbb{F}^{d}\right) & \longrightarrow \mathbb{F}^{n} \\
\varphi & \longmapsto\left(Q\left(\varphi\left(v_{i}\right)\right)_{i=1}^{n} .\right.
\end{array}
$$

The following instance of the hyperplane-marking matroid generalizes the notion of the $d$-parallel matroid of a graph (see $(3))$. Denote by $(d-1) M$ the matroid whose ground set consists of $d-1$ copies of each vector in $E$. The $d$-parallel matrix of $M$ is defined as $H^{d}((d-1) M)$, and the matroid represented by its rows is the (d-dimensional, generic) parallel matroid $\mathcal{P}^{d}(M):=\mathcal{H}^{d}((d-1) M)$. Part (ii) of Proposition 6.1 leads to an interpretation of the geometric meaning carried by the $d$-parallel matrix:

Corollary 6.3. Let $\psi \in \mathbb{F}^{r} \otimes_{\mathbb{F}} \mathbb{F}^{d} \cong \operatorname{Hom}_{\mathbb{F}}\left(\mathbb{F}^{r}, \mathbb{F}^{d}\right)$. Then $\psi \in \operatorname{ker} P^{d}(M)$ if and only if $(\varphi+\psi)\left(v_{i}\right)$ is parallel to $\varphi\left(v_{i}\right)$ for all $i=1,2, \ldots, n$.

Proof. Since there are $d-1$ copies of the vector $v_{i}$ in $(d-1) M$, there will be $(d-1)$ accompanying normal vectors to $\varphi\left(v_{i}\right)$. Because these normals are chosen with generic coordinates, the only vectors normal to all $d-1$ of them are those parallel to $\varphi\left(v_{i}\right)$. Now apply Proposition 6.1.

As in classical rigidity theory, both $R^{d}(M)$ and $H^{d}(M)$ have certain obvious nullvectors.

Proposition 6.4. Let $\psi \in \mathbb{F}^{r} \otimes_{\mathbb{F}} \mathbb{F}^{d} \cong \operatorname{Hom}_{\mathbb{F}}\left(\mathbb{F}^{r}, \mathbb{F}^{d}\right)$.

(i) Given any skew-symmetric $d \times d$ matrix $\sigma \in \mathbb{F}^{d \times d}$, the map $\sigma \circ \psi$, when identified with a vector in $\mathbb{F}^{r} \otimes \mathbb{F}^{d}$, lies in the nullspace of $R^{d}(M)$.

(ii) The map $\psi$, when identified with a vector in $\mathbb{F}^{r} \otimes \mathbb{F}^{d}$, lies in the nullspace of $H^{d}(M)$.

Proof. Assertion (ii) is immediate from the interpretation of the nullspace of $H^{d}(M)$ given in Proposition 6.1.

To prove (i), we define

$$
S:=\mathbb{Z}[\varphi, \sigma, v] /\left(\sigma_{j i}=-\sigma_{i j}\right),
$$

the polynomial ring in the entries of the matrices $\varphi, \sigma, v_{1}, \ldots, v_{n}$. We wish to show that

$$
R^{d}(M)(\sigma \circ \varphi)=0
$$

in $S$. In fact, we will show by a formal calculation that $2 R^{d}(M)(\sigma \circ \varphi)=0$. Since 2 is a non-zero-divisor in $S$, this will imply that $(20)$ holds in $S$, hence remains valid when we pass to $S \otimes_{\mathbb{Z}} \mathbb{F}$ and specialize the entries of $v_{1}, \ldots, v_{n}, \sigma$ to elements of $\mathbb{F}$. 
The calculation ${ }^{3}$ actually takes place in $S[\epsilon] /\left(\epsilon^{2}\right)$. Since $\sigma^{T}=-\sigma$, one has for all $x \in \mathbb{F}^{d}$

$$
\begin{aligned}
Q\left(\left(I_{d}+\epsilon \sigma\right)(x)\right) & =Q(x)+\epsilon\langle x, \sigma(x)\rangle+\epsilon\langle\sigma(x), x\rangle+\epsilon^{2} Q(\sigma(x)) \\
& =Q(x)+\epsilon\left(\langle x, \sigma(x)\rangle+\left\langle x, \sigma^{T}(x)\right\rangle\right)+\epsilon^{2} Q(\sigma(x)) \\
& \equiv Q(x) \quad \bmod \epsilon^{2}
\end{aligned}
$$

Taking $x=\varphi\left(v_{i}\right)$, the function $f$ defined by $f(\varphi):=Q\left(\varphi\left(v_{i}\right)\right)$ has the property

$$
f(\varphi+\epsilon \sigma \circ \varphi) \equiv f(\varphi) \bmod \epsilon^{2} .
$$

On the other hand, expanding $f$ as a Taylor polynomial yields

$$
f(\varphi+\epsilon \sigma \circ \varphi) \equiv f(\varphi)+\epsilon\left\langle\nabla_{\varphi}(f), \sigma \circ \varphi\right\rangle \bmod \epsilon^{2} .
$$

where $\nabla_{\varphi}(f)$ is the gradient of $f$ with respect to the entries of $\varphi$. Therefore $\left\langle\nabla_{\varphi}(f), \sigma \circ \varphi\right\rangle=0$. On the other hand, by Remark 6.2, the $i^{t h}$ row of $R^{d}(M)$ is exactly $\frac{1}{2} \nabla_{\varphi}(f)$. So $2 R^{d}(M) \sigma \circ \varphi=0$ as desired.

6.2. The Nesting Theorem. We have arrived at one of the main results of the paper, the Nesting Theorem, which explains the relationship between the various independence systems associated to an arbitrary representable matroid $M$. In the special case that $M$ is graphic and the ambient dimension $d$ is 2 , the Nesting Theorem gives what we have called the planar trinity (Corollary 6.6 below).

Theorem 6.5 (The Nesting Theorem). Let $M$ be a matroid represented by vectors $E=\left\{v_{1}, \ldots, v_{n}\right\} \subset \mathbb{F}^{r}$, and let $d>1$ be an integer. Then

$$
\mathcal{S}^{1, d}(M) \subseteq \mathcal{R}^{d}(M) \subseteq \mathcal{L}^{d}(M)=\mathcal{H}^{d}(M) \quad\left(=\mathcal{S}^{d-1, d}(M)\right) .
$$

Proof. We first prove that $\mathcal{R}^{d}(M) \subseteq \mathcal{L}^{d}(M)$. It suffices to show that whenever $d \cdot r(M) \leq n$, there is an $\mathbb{F}(\varphi)$-linear dependence among the vectors

$$
\left\{v_{i} \otimes \varphi\left(v_{i}\right)\right\}_{i=1}^{n} \subset \mathbb{F}^{r} \otimes_{\mathbb{F}} \mathbb{F}^{d}
$$

that form the $n$ rows of $R^{d}(M)$. Since $E$ spans a subspace of $\mathbb{F}^{r}$ isomorphic to $\mathbb{F}^{r(M)}$, the rows of $R^{d}(M)$ actually lie in a subspace of dimension $d \cdot r(M)$. If $d \cdot r(M)<n$, then the desired linear dependence is immediate. On the other hand, if $d \cdot r(M)=n$, then Proposition 6.4 implies that the rows of $R^{d}(M)$ lie in a proper subspace of $\mathbb{F}^{r(M)} \otimes \mathbb{F}^{d}$, hence are linearly dependent.

If we replace $v_{i} \otimes \varphi\left(v_{i}\right)$ with $v_{i} \otimes \eta_{i}$, the same argument shows that $\mathcal{H}^{d}(M) \subseteq \mathcal{L}^{d}(M)$.

Next we prove that $\mathcal{S}^{1, d}(M) \subseteq \mathcal{R}^{d}(M)$. Assume that the rows of $R^{d}(M)$ are dependent; we will show that $M$ is $(k, d)$-slope dependent for $k=1$.

We begin with the observation that

$$
\mathcal{S}^{k, d}(M)=\mathcal{L}^{\frac{d}{d-k}}(M) \subseteq \mathcal{L}^{d}(M) .
$$

The equality is Corollary 4.4, and the inclusion follows from the definition of $\mathcal{L}^{m}(M)$ (because $\frac{d}{d-k} \leq d$ ). In particular, if $M$ is $d$-Laman dependent then $M$ is automatically $(k, d)$-slope dependent; we may therefore assume that $M$ is $d$-Laman independent. Without loss of generality, $d \cdot r(M) \geq n$, so the dependence of the rows of $R^{d}(M)$ implies the vanishing of every one of its $n \times n$ minor subdeterminants. Moreover, by Theorem 3.6, $M$ admits a $d$-Edmonds decomposition (see Definition 3.5). Associating the vectors $v_{1}, \ldots, v_{n}$ with their indices $[n]=\{1, \ldots, n\}$, we may write this Edmonds decomposition concisely as $[n]=\bigsqcup_{j=1}^{d} I_{j}$.

Claim. There exists an $n \times n$ minor $\xi$ of $R^{d}(M)$ that is a nonzero multihomogeneous polynomial in the coordinates of the vectors $\varphi\left(v_{i}\right)$.

Given the claim, if $\xi$ vanishes on the non-annihilating cellule $X_{k, d}^{\varnothing}(M)$ of the photo space, then the projection on $X_{k, d}^{\varnothing}(M) \rightarrow \mathbb{G} r\left(k, \mathbb{F}^{d}\right)$ is not Zariski dense, because the homogeneous coordinates of the $\varphi\left(v_{i}\right)$ are in fact the Plücker coordinates on $\mathbb{G} r\left(k, \mathbb{F}^{d}\right)$. Hence by Theorem 4.3, the claim is all we need for the present theorem.

\footnotetext{
${ }^{3}$ This calculation is identical to that usually used to show that the orthogonal group with respect to the quadratic form $Q$ on $\mathbb{F}^{d}$ has its Lie algebra equal to the space of skew-symmetric matrices.
} 
Let $x^{(i)}:=\varphi\left(v_{i}\right)$, and let $v_{i}=\left[v_{i 1} \cdots v_{i r}\right]^{T}$. Group the columns of $R=R^{d}(M)$ in blocks, so that the $i^{t h}$ row of $R$ is

$$
\left[v_{i 1} x_{1}^{(i)} \cdots v_{i r} x_{1}^{(i)}\left|v_{i 1} x_{2}^{(i)} \cdots v_{i r} x_{2}^{(i)}\right| \cdots \mid v_{i 1} x_{d}^{(i)} \cdots v_{i r} x_{d}^{(i)}\right] .
$$

Each $n \times n$ submatrix $R_{A}$ of $R$ is indexed by some choice of an $n$-element subset $A$ of the $d r$ columns. Letting $A_{i}$ be the subset of $A$ coming from columns in the $i^{\text {th }}$ block, one obtains a sequence of subsets $A_{1}, \ldots, A_{d} \subset[r]$ with $n=|A|=\sum_{j=1}^{d}\left|A_{j}\right|$. Then

$$
\operatorname{det} R_{A}=\sum_{I} \varepsilon(I) \sum_{\sigma_{1}, \ldots, \sigma_{d}} \varepsilon\left(\sigma_{1}\right) \ldots \varepsilon\left(\sigma_{d}\right) \prod_{j=1}^{d} \prod_{i \in I_{j}} v_{i, \sigma_{j}(i)} x_{j}^{(i)} .
$$

Here the first sum ranges over all partitions $I=\left\{I_{1}, \ldots, I_{d}\right\}$ of $[n]$ with $d$ parts, the second sum ranges over all $d$-tuples of bijections $\sigma_{j}: I_{j} \rightarrow A_{j}$, and $\varepsilon(C), \varepsilon\left(\sigma_{j}\right) \in\{ \pm 1\}$ (there are explicit formulas for these signs, but we won't need them). This expression may be simplified:

$$
\begin{aligned}
\operatorname{det} R_{A} & =\sum_{I} \varepsilon(I)\left(\prod_{j=1}^{d} \prod_{i \in I_{j}} x_{j}^{(i)}\right)\left(\prod_{j=1}^{d} \sum_{\sigma_{j}: I_{j} \rightarrow A_{j}} \varepsilon\left(\sigma_{j}\right) v_{i, \sigma_{j}(i)}\right) \\
& =\sum_{I}\left(\prod_{j=1}^{d} \prod_{i \in I_{j}} x_{j}^{(i)}\right)\left(\varepsilon(I) \prod_{j=1}^{d} \operatorname{det} V_{I_{j}, A_{j}}\right)
\end{aligned}
$$

where $V_{I_{j}, A_{j}}$ is the submatrix of $\left[v_{i k}\right]_{i=1, \ldots, n, k=1, \ldots, r}$ with rows $I_{j}$ and columns $A_{j}$. Note $\operatorname{that} \operatorname{det}\left(V_{I_{j}, A_{j}}\right) \in \mathbb{F}$, so the calculation implies that $\operatorname{det} R_{A}$ is a multihomogeneous polynomial in the coordinates $\left\{x_{j}^{(i)}\right\}$ with coefficients in $\mathbb{F}$.

By the definition of an Edmonds decomposition, the sets $I_{1}, \ldots, I_{d}$ are independent in $M$. Hence there is some subset $A_{j} \subseteq[r]$ with $\operatorname{det} V_{I_{j}, A_{j}} \neq 0$. The monomial corresponding to this choice of $I_{j}$ 's and $A_{j}$ 's has a nonzero coefficient in the multihomogeneous polynomial $\xi=\operatorname{det} R_{A}$. Therefore $\xi \neq 0$, establishing the claim and completing the proof that $\mathcal{S}^{1, d}(M) \subseteq \mathcal{R}^{d}(M)$.

Replacing $R^{d}(M)$ with $H^{d}(M), k=1$ with $k=d-1$, and $\varphi\left(v_{i}\right)$ with $\eta_{i}$ throughout, the same argument shows that $\mathcal{S}^{d-1, d} \subseteq \mathcal{H}^{d}(M)$. Since $\mathcal{S}^{d-1, d}(M)=\mathcal{L}^{d}(M)$ by Corollary 4.4, we are done.

The case $d=2$ is very special. Recall that $\mathcal{P}^{d}(M)=\mathcal{H}^{d}((d-1) M)$, so $\mathcal{P}^{2}(M)=\mathcal{H}^{2}(M)$. Indeed, the Nesting Theorem implies much more:

Corollary 6.6. Let $M$ be a matroid represented as above. Then

$$
\mathcal{S}^{1,2}(M)=\mathcal{R}^{2}(M)=\mathcal{L}^{2}(M)=\mathcal{H}^{2}(M)=\mathcal{P}^{2}(M) .
$$

Remark 6.7. Setting $d=1$ collapses the Nesting Theorem to

$$
\mathcal{S}^{k, \infty}(M)=\mathcal{R}^{1}(M)=\mathcal{L}^{1}(M)=M
$$

However, these phenomena are somewhat more trivial. To make sense of the complexes $\mathcal{S}^{k, \infty}(M)$ and $\mathcal{L}^{1}(M)$, consider the identity $\mathcal{S}^{k, d}(M)=\mathcal{L}^{\frac{d}{d-k}}(M)$ of Corollary 4.4. Fixing $k$ and letting $d \rightarrow \infty($ as a positive integer), we obtain $\mathcal{S}^{k, \infty}(M)=\mathcal{R}^{1}(M)=\mathcal{L}^{1}(M)$. On the other hand, it is an easy consequence of the definitions of $\mathcal{L}^{m}(N)$ and $\mathcal{R}^{d}(M)$ that $\lim _{m \rightarrow 1^{+}} \mathcal{L}^{m}(M)=M=\mathcal{R}^{1}(M)$.

Remark 6.8. There is in fact a simple explicit isomorphism between the matroids $\mathcal{R}^{2}(M)$ and $\mathcal{H}^{2}(M)$ $\left(=\mathcal{P}^{2}(M)\right)$. Let $\rho$ be the " $\pi / 2$ rotation" $\mathbb{F}^{2} \rightarrow \mathbb{F}^{2}$ given by

$$
\left[\begin{array}{cc}
0 & -1 \\
1 & 0
\end{array}\right]
$$

Then $\rho\left(\varphi\left(v_{i}\right)\right)=\eta_{i}$, a generic normal to the generic image vector $\varphi\left(v_{i}\right)$, and the invertible linear operator $1_{\mathbb{F}^{r}} \otimes \rho$ on $\mathbb{F}^{r} \otimes_{\mathbb{F}} \mathbb{F}^{d}$ sends $v_{i} \otimes \varphi\left(v_{i}\right)$ to $v_{i} \otimes \eta_{i}$. 
Remark 6.9. When $d \geq 3$, the inclusion $\mathcal{R}^{d}(M) \subseteq \mathcal{L}^{d}(M)$ is usually strict. By Proposition 6.4, the nullspace of $R^{d}(M)$ contains the $\left(\begin{array}{l}d \\ 2\end{array}\right)$-dimensional space of all vectors of the form $\sigma \circ \varphi$, as $\sigma$ ranges over all skewsymmetric matrices in $\mathbb{F}^{d \times d}$. Consequently, every $d$-rigidity-independent subset $A \subseteq E$ must satisfy $|A| \leq$ $d \cdot r(A)-\left(\begin{array}{l}d \\ 2\end{array}\right)$. On the other hand, there may exist $d$-Laman independent sets $A$ of cardinality up to $d \cdot r(A)-1$.

\section{EXAMPLES: UNIFORM MATROIDS}

Let $E$ be a ground set with $n$ elements. The uniform matroid of rank $r$ on $E$ is defined to be the matroid whose independent sets are

$$
U_{r, n}=\{F \subseteq E:|F| \leq r\} .
$$

Broadly speaking, $U_{r, n}$ can be regarded as the matroid represented by $n$ generically chosen vectors in $\mathbb{F}^{r}$, where $\mathbb{F}$ is a sufficiently large field.

Predictably, the $d$-Laman independence complex on $U_{r, n}$ is also a uniform matroid for every $d$. More surprising is that $d$-Laman independence carries nontrivial geometric information about sets of $n$ generic vectors in $r$-space - specifically coplanarity for $U_{2,3}$ and the cross-ratio for $U_{2,4}$.

Proposition 7.1. Let $U_{r, n}$ be the uniform matroid of rank $r$ on $n$ elements, and let $d \in(1, \infty)_{\mathbb{R}}$. Then

$$
\mathcal{L}^{d}\left(U_{r, n}\right)=U_{s, n} \quad \text { where } s=\min (\lceil d r-1\rceil, n) .
$$

and

$$
\mathcal{S}^{k, d}\left(U_{r, n}\right)=U_{t, n} \quad \text { where } t=\min \left(\left\lceil\frac{d r}{d-k}-1\right\rceil, n\right) .
$$

Proof. We know that $\mathcal{L}^{d}\left(U_{r, n}\right)$ is a simplicial complex, and it is easy to see that the criteria for $F$ to be $d$-Laman independent can depend only depend on the cardinality $|F|$. Therefore

$$
\begin{aligned}
\mathcal{L}^{d}\left(U_{r, n}\right) & =\left\{F \subseteq E: d \cdot r\left(F^{\prime}\right)>\left|F^{\prime}\right| \text { for all nonempty } F^{\prime} \subseteq F\right\} \\
& =\{F \subseteq E: d \cdot r(F)>|F|\} \\
& =\{F \subseteq E:|F|<d r\} \\
& =U_{s, n},
\end{aligned}
$$

which is (21). Applying Corollary 4.4 to (21) gives (22).

Example 7.2 $\left(U_{2,3}\right)$. Let $\mathbb{F}$ be any field, and let $e_{1}, e_{2}$ be the standard basis vectors in $\mathbb{F}^{2}$. The matroid $M=U_{2,3}$ is represented by the vectors $\left\{e_{1}, e_{1}+e_{2}, e_{2}\right\} \subset \mathbb{F}^{2}$; this representation is unique up to the action of the projective general linear group. By Proposition 7.1,

$$
\mathcal{L}^{d}\left(U_{2,3}\right)=\left\{\begin{array}{ll}
U_{2,3} & \text { if } d \in\left(1, \frac{3}{2}\right]_{\mathbb{R}} \\
U_{3,3} & \text { if } d \in\left(\frac{3}{2}, \infty\right)_{\mathbb{R}}
\end{array} \quad \text { and } \quad \mathcal{S}^{1, d}\left(U_{2,3}\right)= \begin{cases}U_{3,3} & \text { if } d=2 \\
U_{2,3} & \text { if } d \in\{3,4, \ldots\}\end{cases}\right.
$$

We now consider what these equalities mean in terms of slopes. Let $\varphi: \mathbb{F}^{2} \rightarrow \mathbb{F}^{d}$ be a linear transformation. If $d=2$, then the images $\varphi\left(e_{1}\right), \varphi\left(e_{1}+e_{2}\right), \varphi\left(e_{2}\right)$ can have arbitrary slopes as $\varphi$ varies. This is why $\mathcal{S}^{1,2}\left(U_{2,3}\right)=U_{3,3}$. On the other hand, when $d \geq 3$, those three vectors must be coplanar. This imposes a nontrivial constraint on the homogeneous coordinates for the lines spanned by the three images, and explains why $\mathcal{S}^{1, d}\left(U_{2,3}\right)=U_{2,3}$.

By direct calculation, the vectors

$$
e_{1} \otimes \varphi\left(e_{1}\right), \quad\left(e_{1}+e_{2}\right) \otimes \varphi\left(e_{1}+e_{2}\right), \quad e_{2} \otimes \varphi\left(e_{2}\right)
$$

are linearly dependent if and only if $d=1$. Therefore

$$
\mathcal{R}^{d}\left(U_{2,3}\right)= \begin{cases}U_{2,3} & \text { if } d=1 \\ U_{3,3} & \text { if } d \in\{2,3, \ldots\} .\end{cases}
$$

In this case, the inclusions $\mathcal{R}^{d}(M) \subseteq \mathcal{L}^{d}(M)$ given by Theorem 6.5 turn out to be equalities. 
Example 7.3 $\left(U_{2,4}\right)$. Let $\mathbb{F}$ be a field of cardinality $>2$, let $\mu \in \mathbb{F} \backslash\{0,1\}$, and let $e_{1}, e_{2}$ be the standard basis vectors in $\mathbb{F}^{2}$. The four vectors

$$
\left\{e_{1}, e_{1}+e_{2}, \quad e_{2}, \quad e_{1}+\mu e_{2}\right\}
$$

represent $M=U_{2,4}$ over $\mathbb{F}$. Again, this representation is unique up to projective equivalence. By Proposition 7.1 ,

$$
\mathcal{L}^{d}\left(U_{2,4}\right)=\left\{\begin{array}{ll}
U_{2,4} & \text { if } d \in\left(1, \frac{3}{2}\right]_{\mathbb{R}} \\
U_{3,4} & \text { if } d \in\left(\frac{3}{2}, 2\right]_{\mathbb{R}} \\
U_{4,4} & \text { if } d \in(2, \infty)_{\mathbb{R}}
\end{array} \quad \text { and } \quad \mathcal{S}^{1, d}\left(U_{2,4}\right)= \begin{cases}U_{3,4} & \text { if } d=2 \\
U_{2,4} & \text { if } d \in\{3,4, \ldots\} .\end{cases}\right.
$$

Why is this correct from the point of view of slopes? From Example 7.2, we know that when $d \geq 3$, the lines spanned by the images of any three of the four vectors must be coplanar, so there is an algebraic dependence among the homogeneous coordinates for these three lines. For $d=2$, this does not happen; the slopes of the images of any triple can be made arbitrary. However, applying a linear transformation to the representing vectors does not change their cross-ratio (in this case $\mu$ ), so the fourth image vector is determined by the first three. This is the geometric interpretation of the combinatorial identity $\mathcal{S}^{1,2}\left(U_{2,4}\right)=U_{3,4}$.

Direct calculation shows that every three of the four vectors

$$
w_{1}:=e_{1} \otimes \varphi\left(e_{1}\right), \quad w_{2}:=\left(e_{1}+e_{2}\right) \otimes \varphi\left(e_{1}+e_{2}\right), \quad w_{3}:=e_{2} \otimes \varphi\left(e_{2}\right), \quad w_{4}:=\left(e_{1}+\mu e_{2}\right) \otimes \varphi\left(e_{1}+\mu e_{2}\right)
$$

are linearly dependent when $d=1$, but independent for all $d \geq 2$. When $d \geq 2$, there is an additional, less obvious linear dependence: $(\mu-1) w_{1}-\mu w_{2}+\left(\mu-\mu^{2}\right) w_{3}+w_{4}=0$. Consequently

$$
\mathcal{R}^{d}\left(U_{2,4}\right)= \begin{cases}U_{2,4} & \text { if } d=1, \\ U_{3,4} & \text { if } d \in\{2,3, \ldots\} .\end{cases}
$$

This calculation is independent of the particular coordinates chosen for the representing vectors, even up to projective equivalence (that is, up to the choice of the parameter $\mu$ ): that is, $\mathcal{R}^{d}\left(U_{2,4}\right)$ is a combinatorial invariant.

On the other hand, unlike the situation for $U_{2,3}$, the inclusions $\mathcal{R}^{d}(M) \subseteq \mathcal{L}^{d}(M)$ given by Theorem 6.5 turn out to be strict. In particular, $\mathcal{R}^{\infty}(M)$ is not Boolean while $\mathcal{L}^{\infty}(M)$ is always Boolean. This behavior deviates notably from the case of graphic matroids (see Proposition 8.5 below).

\section{More on $\mathcal{R}^{d}(M)$ : INVARIANCE AND STABILIZATION}

The examples in the previous section raise some natural questions. Clearly $\mathcal{L}^{m}(M)$ is a combinatorial invariant of $M$, that is, it does not depend on the choice of representation, nor the field of representation. Hence by Corollary 4.4, the same is true for $\mathcal{S}^{k, d}(M)$, and in particular $\mathcal{H}^{d}(M)$ and $\mathcal{P}^{d}(M)$. But what about $\mathcal{R}^{d}(M)$ ? This is an issue which does not arise in classical rigidity theory, as the graphic matroid $M(G)$ is always represented by the vectors

$$
\left\{e_{i}-e_{j}:\{i, j\} \in E(G)\right\}
$$

where $e_{i}$ is the $i^{t h}$ standard basis vector in $\mathbb{R}^{|V(G)|}$. In fact, Proposition 8.1 below will show that $\mathcal{R}^{d}(M)$ is a projective invariant of a matroid represented over a given field. A result of $\mathrm{N}$. White shows that graphic matroids, and more generally matroids that can be represented over $\mathbb{F}_{2}$, are projectively unique when represented over any fixed field; see, e.g., [18, Proposition 1.2.5]. It will follow that $\mathcal{R}^{d}(M(G))$ is a combinatorial invariant of a graphic matroid $M(G)$ over any fixed field.

We begin by recalling the notion of projective equivalence for representations of a matroid. Two sets of vectors $E=\left\{v_{1}, \ldots, v_{n}\right\}, E^{\prime}=\left\{v_{1}^{\prime}, \ldots, v_{n}^{\prime}\right\} \subset \mathbb{F}^{r}$ are called projectively equivalent if there are nonzero scalars $c_{1}, \ldots, c_{n} \in \mathbb{F}^{\times}$and an invertible linear transformation $g \in G L_{r}(\mathbb{F})$, such that $v_{i}^{\prime}=g\left(c_{i} v_{i}\right)$ for every $i$. It is easy to see that in this case, the matroids represented by $E$ and $E^{\prime}$ are combinatorially identical. As we now show, the same is true for their $d$-rigidity matroids.

Proposition 8.1. Let $M, M^{\prime}$ be matroids represented by projectively equivalent sets $E, E^{\prime} \subset \mathbb{F}^{r}$, and let $d \geq 2$. Then $\mathcal{R}^{d}(M)=\mathcal{R}^{d}\left(M^{\prime}\right)$. 
Proof. For $v \in E$ and $c \in \mathbb{F}^{\times}$, replacing $v$ with $c v$ has the effect of multiplying $v \otimes \varphi(v)$ by $c^{2}$, which does not change the matroid $\mathcal{R}^{d}(M)$.

For the second assertion, let $g \in G L_{r}(\mathbb{F})$, and suppose that we have an $\mathbb{F}(\varphi)$-linear dependence

$$
\sum_{i=1}^{n} c_{i} v_{i} \otimes v^{(i)}=0
$$

in $\mathbb{F}^{r} \otimes \mathbb{F}(\varphi)^{d}$. The group $G L_{r}(\mathbb{F})$ acts $\mathbb{F}(\varphi)$-linearly on $\mathbb{F}^{r} \otimes \mathbb{F}(\varphi)^{d}$ by $g(v \otimes w)=g(v) \otimes w$. Applying $g$ to (24) yields

$$
\sum_{i=1}^{n} c_{i} g\left(v_{i}\right) \otimes v^{(i)}=g(0)=0 .
$$

Equivalently,

$$
\sum_{i=1}^{n} c_{i} g\left(v_{i}\right) \otimes\left(\varphi \circ g^{-1}\right) g\left(v_{i}\right)=0 .
$$

The entries of the $d \times r$ matrix $\varphi \circ g^{-1}$ are algebraically independent transcendentals over $\mathbb{F}$ (because $\varphi$ was), and the transcendental extensions $\mathbb{F}(\varphi)$ and $\mathbb{F}\left(\varphi \circ g^{-1}\right)$ coincide because $g$ is invertible. Hence the matroid represented by $\left\{g\left(v_{1}\right), \ldots, g\left(v_{n}\right)\right\}$ contains the same dependence $(24)$ as do $\left\{v_{1}, \ldots, v_{n}\right\}$. Considering all such dependences and replacing $g$ with $g^{-1}$, one sees that this matroid is combinatorially identical to $\mathcal{R}^{d}(M)$.

Question 8.2. Is $\mathcal{R}^{d}(M)$ a combinatorial invariant of $M$, or does it depend on the choice of field $\mathbb{F}$ and the particular representation $\left\{v_{1}, \ldots, v_{n}\right\}$ of $M$ in $\mathbb{F}^{r}$ ?

In the special case $d=2$, the Nesting Theorem implies that $\mathcal{R}^{d}(M)$ is indeed a combinatorial invariant. While we have no reason to expect invariance in all cases, we have not found a counterexample. We have seen that $\mathcal{R}^{d}(M)$ is indeed combinatorial when $M=U_{2,3}$ or $U_{2,4}$. In what follows, we describe a matroid with two projectively inequivalent representations whose $d$-rigidity matroids coincide.

Example 8.3. Consider the following two sets of nine coplanar vectors in $\mathbb{R}^{3}$ :

$$
\begin{aligned}
E & =\{(1,0,0),(1,0,1),(1,0,2),(1,1,0),(1,1,1),(1,1,2),(1,2,0),(1,2,1),(1,2,2)\}, \\
E^{\prime} & =\{(1,0,0),(1,0,1),(1,0,3),(1,2,0),(1,2,1),(1,2,3),(1,3,0),(1,4,1),(1,6,3)\} .
\end{aligned}
$$
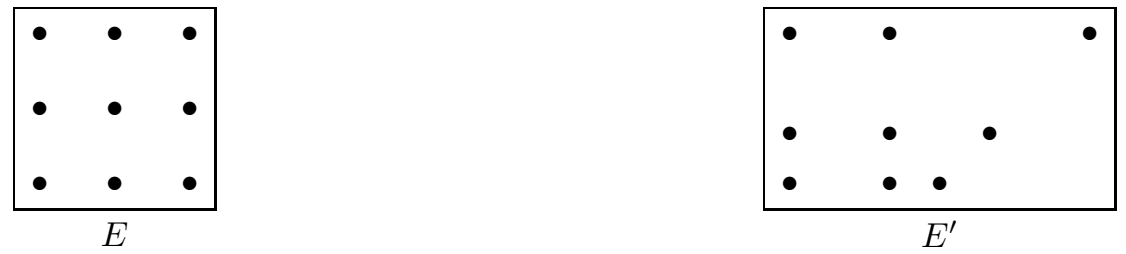

Let $M, M^{\prime}$ be the matroids represented by $E, E^{\prime}$ respectively. These matroids are combinatorially isomorphic, but $E$ and $E^{\prime}$ are certainly projectively inequivalent. On the other hand, computations using Mathematica show that $\mathcal{R}^{2}(M)=\mathcal{R}^{2}\left(M^{\prime}\right)\left(=U_{5,9}\right)$ and that $\mathcal{R}^{3}(M)=\mathcal{R}^{3}\left(M^{\prime}\right)$ (the bases are the subsets of $E$ (resp. $E^{\prime}$ ) of cardinality 6 , except for the complements of the eight affine lines.)

We next discuss how $\mathcal{R}^{d}(M)$ stabilizes for large $d$. Let $\omega: \mathbb{F}\left(\varphi_{1,1}, \ldots, \varphi_{d+1, r}\right) \rightarrow \mathbb{F}\left(\varphi_{1,1}, \ldots, \varphi_{d, r}\right)$ be the map sending $\varphi_{d+1, j}$ to 0 for every $j$. Then $\omega$ takes linear dependences on rows of $\mathcal{R}^{d+1}(M)$ to linear dependences on rows of $\mathcal{R}^{d}(M)$. Therefore $\mathcal{R}^{d}(M) \subseteq \mathcal{R}^{d+1}(M)$.

Since there are only finitely many simplicial complexes on a fixed finite ground set $E$, the tower

$$
M=\mathcal{R}^{1}(M) \subseteq \mathcal{R}^{2}(M) \subseteq \mathcal{R}^{3}(M) \subseteq \cdots
$$

must eventually stabilize to some complex $\mathcal{R}^{\infty}(M)$. We can say more precisely when this stabilization occurs.

Proposition 8.4. Let $M$ be a matroid represented by $E=\left\{v_{1}, \ldots, v_{n}\right\} \subset \mathbb{F}^{r}$, where (without loss of generality) $M$ has rank $r$. Then for every $d \geq r$,

$$
\mathcal{R}^{d}(M)=\mathcal{R}^{r}(M)=\mathcal{R}^{\infty}(M) .
$$


Proof. Since $\mathcal{R}^{d}(M) \subseteq \mathcal{R}^{d+1}(M)$, it suffices to prove that $\mathcal{R}^{d}(M) \subseteq \mathcal{R}^{r}(M)$ for $d \geq r$. Let $\varphi$ be an $r \times r$ matrix of transcendentals over $\mathbb{F}$. Suppose that we have a linear dependence of the form (24). Let $\psi$ be another $d \times r$ matrix of transcendentals, so that $\mathbb{F}(\varphi) \hookrightarrow \mathbb{F}(\varphi, \psi)$ is a purely transcendental extension. Viewing the matrix $\psi$ as a $\mathbb{F}(\varphi, \psi)$-linear map, one can apply it to the second factor of $\mathbb{F}^{r} \otimes \mathbb{F}(\varphi)^{r}$. Applying this to (24) gives

$$
\sum_{i=1}^{n} c_{i} v_{i} \otimes(\psi \circ \varphi)\left(v_{i}\right)=0
$$

which is an $\mathbb{F}(\varphi, \psi)$-linear dependence on the vectors $\left\{v_{i} \otimes(\psi \circ \varphi)\left(v_{i}\right)\right\}_{i=1, \ldots, n}$.

We claim that $\mathbb{F}(\varphi, \psi)$ is purely transcendental over $\mathbb{F}(\psi \circ \varphi)$. To see this, first note that $\mathbb{F}(\psi, \varphi)=$ $\mathbb{F}\left(\psi \circ \varphi, \varphi^{-1}\right)$. That is, $\mathbb{F}(\psi, \varphi)$ can be obtained from $\mathbb{F}(\psi \circ \varphi)$ by adjoining $r^{2}$ elements, namely the entries of $\varphi^{-1}$. In particular, the transcendence degree of $\mathbb{F}(\psi, \varphi)$ over $\mathbb{F}(\psi \circ \varphi)$ is at most $r^{2}$. Similarly, the transcendence degree of $\mathbb{F}(\psi \circ \varphi)$ over $\mathbb{F}$ is at most $d r$. But $\mathbb{F}(\psi, \varphi)$ clearly has transcendence degree $d r+r^{2}$ over $\mathbb{F}$, and transcendence degree is additive in towers of field extensions [5, Thm. VI.1.11], so both instances of "at most" may be replaced with "exactly", proving the claim.

By the existence of the $\mathbb{F}(\varphi, \psi)$-linear dependence (25), we conclude that the vectors $\left\{v_{i} \otimes(\psi \circ \varphi)\left(v_{i}\right)\right\}_{i=1, \ldots, n}$ must also be $\mathbb{F}(\psi \circ \varphi)$-linearly dependent. Therefore $\mathcal{R}^{d}(M) \subseteq \mathcal{R}^{r}(M)$ as desired.

When a matroid $M$ can be represented over different fields, it is natural to ask how much $\mathcal{R}^{d}(M)$ can vary. For instance, if $M=M(G)$ is graphic, then the standard representation (23) is valid over every field $\mathbb{F}$ and unique up to projective equivalence once the field is fixed, as mentioned earlier. For sufficiently large $d$, the $d$-rigidity matroid of $M(G)$ is also independent of the choice of the field $\mathbb{F}$, as we now explain.

Proposition 8.5. Let $M=M(G)$ be the graphic matroid representing an n-vertex graph $G$ over an arbitrary field $\mathbb{F}$, equipped with the standard representation (23). Then $\mathcal{R}^{n}(M)=2^{E}=\mathcal{R}^{\infty}(M)$.

Proof. Let $K_{n}$ be the complete graph on $n$ vertices. Since $R^{n}(M)$ is a row-selected submatrix of $R^{n}\left(M\left(K_{n}\right)\right)$, it suffices to assume that $G=K_{n}$.

To avoid overly cumbersome notation, we give the proof for $n=4$; the argument for arbitrary $n$ should be clear from this case. For $n=4$, the $6 \times 12$ rigidity matrix $R^{4}\left(M\left(K_{4}\right)\right.$ ) is as follows. (Each nonzero entry is a binomial $\varphi_{i j}-\varphi_{i k}$, written on two lines so that the matrix is not too wide for the page.)

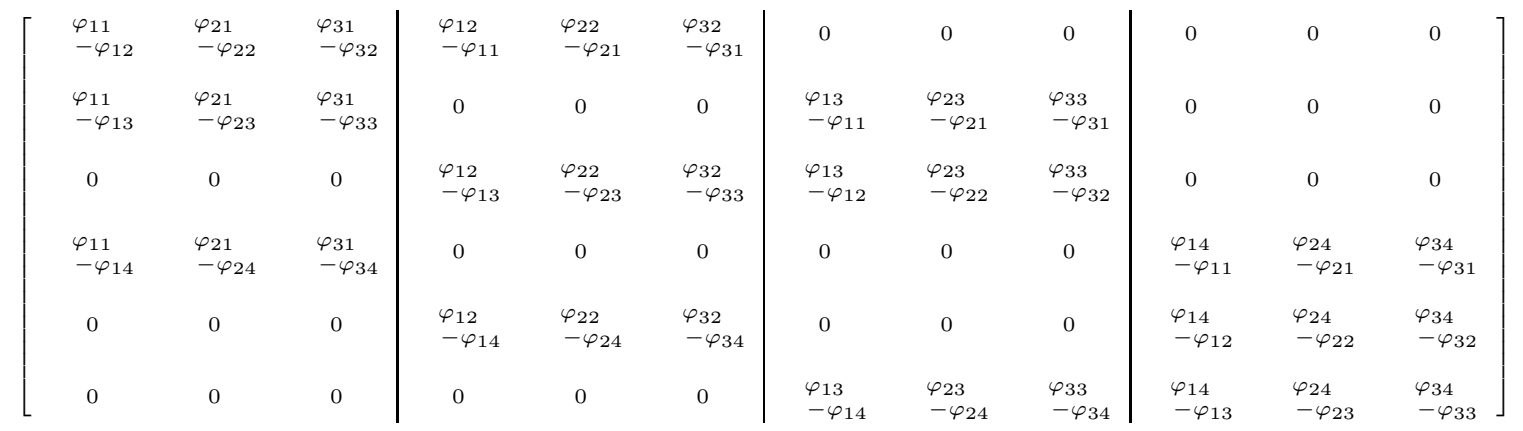

We must show that some $6 \times 6$ minor of $R^{4}\left(M\left(K_{4}\right)\right)$ is nonsingular. Consider the submatrix $M^{\prime}$ consisting of the last column in the second block, the last two columns in the third block, and all three columns in the fourth block:

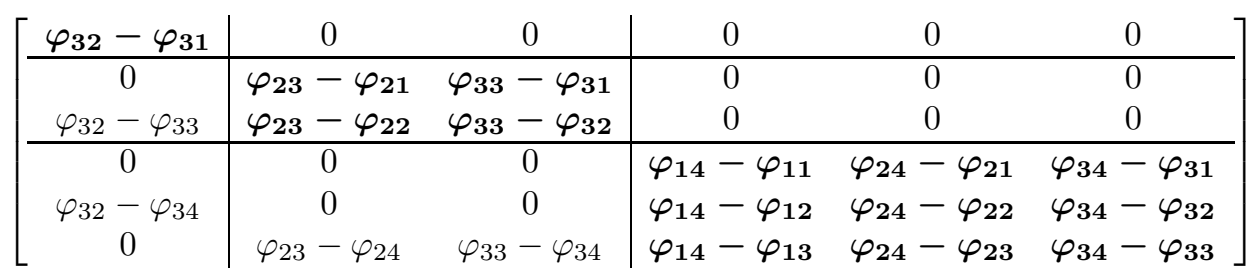

Since $M^{\prime}$ is block lower triangular, its determinant is the product of the determinants of the blocks along the diagonal (indicated in boldface). Each such determinant is a nonzero polynomial in the $\varphi_{i j}$ over any 
field, because the coefficients of $\varphi_{31}$ in the first block, $\varphi_{21} \varphi_{32}$ in the second block, and $\varphi_{11} \varphi_{22} \varphi_{33}$ in the third block are all \pm 1 . Therefore $M^{\prime}$ is nonsingular over any field, as desired.

This observation begs the question of whether $\mathcal{R}^{d}(M(G))$ depends on the field before $d$ reaches the stable range. For an arbitrary representable matroid $M$, it is not true in general that $\mathcal{R}^{\infty}(M)$ is Boolean. We have already seen one example for which this fails, namely $U_{2,4}$. Another example is the well-known Fano matroid $F$, represented over the two-element field $\mathbb{F}_{2}$ by the seven nonzero elements of $\mathbb{F}_{2}^{3}$. It is not hard to show that $\mathcal{L}^{d}(F)$ is Boolean for $d>\frac{7}{3}$. On the other hand, computation with Mathematica indicates that $\mathcal{R}^{2}(F)=U_{5,7}$, but $\mathcal{R}^{d}(F)=U_{6,7}$ for all integers $d \geq 3$.

\section{Open PROBLEMS}

The foregoing results raise many questions that we think are worthy of further study; some of these have been mentioned earlier in the paper. In this final section, we restate the open problems and add a few more.

Problem 1. Determine the singular locus of the $(k, d)$-photo space $X_{k, d}(M)$ (perhaps by calculating the dimension of its various tangent spaces, as in Proposition 4.8).

Problem 2. Give a direct combinatorial explanation for Corollary 5.3, presumably by identifying some natural relationship between photos of $M$ and of $M^{\perp}$.

Problem 3. Explain the "scaling phenomenon" of Remark 4.7 geometrically.

Problem 4. Determine whether or not the $d$-rigidity matroid $\mathcal{R}^{d}(M)$ is a combinatorial invariant of $M$ (Question 8.2). If not, determine which matroids have this property, and to what extent $\mathcal{R}^{d}(M)$ depends on the field $\mathbb{F}$ over which $M$ is represented. In particular, is $\mathcal{R}^{d}(M)$ independent of $\mathbb{F}$ in the case that $M$ is a graphic matroid?

Crapo gave an elegant characterization [19, Theorem 8.2.2] of $\mathcal{H}^{d}(M)$ when $M$ is graphic. A basis of $\mathcal{H}^{d}(M)$ is a (multi-)set of edges having a $(d+1) \mathbf{T} d$-covering, or a decomposition into $d+1$ edge-disjoint trees, exactly $d$ incident with each vertex, with no $d$ nonempty subtrees spanning the same subset of vertices.

Problem 5. Generalize Crapo's characterization of $\mathcal{H}^{d}(M)$ to the case of a non-graphic matroid $M$.

A vertex of a graph $G$ corresponds to a cocircuit of $M(G)$ whose deletion leaves a connected matroid. However, there is no analogous notion of "vertex" when $M$ is a non-graphic matroid (although the foregoing may be helpful if $M$ is sufficiently connected). Similarly, it is unclear how to generalize to non-graphic matroids (and to higher dimensions) other fundamentals of graphic rigidity theory; for instance, Henneberg's construction of the bases for $\mathcal{H}^{2}(M)=\mathcal{R}^{2}(M)=\mathcal{L}^{2}(M)$ [19, Theorem 2.2.3].

Our last open problem is similar in spirit to the results of [7] and [9], describing the algebraic and combinatorial structure of the equations defining the slope variety of a graph. It is motivated also by the appearance of the cross-ratio in Example 7.3.

Problem 6. Describe explicitly the defining equations (in Plücker coordinates on $\left.\mathbb{G} r\left(k, \mathbb{F}^{d}\right)^{n}\right)$ for $\overline{\pi X_{k, d}^{\varnothing}(M)}$, where $\pi$ is the projection map of (7).

\section{ACKNOWLEDGMENTS}

A substantial portion of this work was completed in July 2004 at the Park City Mathematical Institute, sponsored by the Institute for Advanced Study. The authors particularly thank Walter Whiteley for sharing with them some of his manuscripts on classical rigidity theory. They also thank Gil Kalai, Ezra Miller, Neil White and Günter Ziegler for useful discussions, and an anonymous referee for a careful reading and numerous thoughtful suggestions.

\section{REFERENCES}

[1] M. Aigner, Combinatorial Theory. Springer-Verlag, Berlin, 1997.

[2] T. Brylawski and J. Oxley, The Tutte polynomial and its applications, Matroid applications, 123-225, Encyclopedia Math. Appl. 40, Cambridge Univ. Press, Cambridge, 1992. 
[3] J. Edmonds, Minimum partition of a matroid into independent subsets, J. Res. Nat. Bur. Standards Sect. B 69B (1965), 69-72.

[4] J. Graver, B. Servatius, and H. Servatius, Combinatorial rigidity. Graduate Studies in Mathematics, 2. American Mathematical Society, Providence, RI, 1993.

[5] T. Hungerford, Algebra. Graduate Texts in Mathematics, 73. Springer-Verlag, New York, 1974.

[6] G. Laman, On graphs and rigidity of plane skeletal structures, J. Engrg. Math. 4 (1970), 331-340.

[7] J.L. Martin, Geometry of graph varieties, Trans. Amer. Math. Soc. 355 (2003), 4151-4169.

[8] J.L. Martin, On the topology of graph picture spaces, Adv. Math. 191, no. 2 (2005), 312-338.

[9] J.L. Martin, The slopes determined by $n$ points in the plane, Duke Math. J. to appear.

[10] C.St.J.A. Nash-Williams, Edge-disjoint spanning trees of finite graphs, J. London Math. Soc. 36 (1961), 445-450.

[11] C.St.J.A. Nash-Williams, Decompositions of finite graphs into forests, J. London Math. Soc. 39 (1964), 12.

[12] J.G. Oxley, Matroid theory. Oxford Science Publications, The Clarendon Press, Oxford University Press, New York, 1992.

[13] A. Recski, A network theory approach to the rigidity of skeletal structures. II. Laman's theorem and topological formulae. Discrete Appl. Math. 8 (1984), no. 1, 63-68.

[14] R.P. Stanley, Enumerative combinatorics, Vol. I, Cambridge Studies in Advanced Mathematics 49, Cambridge University Press, Cambridge, 1997.

[15] T.-S. Tay, A new proof of Laman's theorem, Graphs Combin. 9 (1993), 365-370.

[16] W.T. Tutte, On the problem of decomposing a graph into $n$ connected factors. J. London Math. Soc. 36 (1961), 221-230.

[17] D.J.A. Welsh, Matroid theory. London Math. Soc. Monographs 8, Academic Press [Harcourt Brace Jovanovich, Publishers], London-New York, 1976

[18] N. White, Coordinatizations, Combinatorial geometries, 1-27, Encyclopedia Math. Appl. 29, Cambridge Univ. Press, Cambridge, 1987.

[19] W. Whiteley, Some matroids from discrete applied geometry, Contemp. Math. 197 (1996), 171-311.

[20] W. Whiteley, Rigidity and scene analysis, Handbook of discrete and computational geometry, 893-916, CRC Press Ser. Discrete Math. Appl., CRC, Boca Raton, FL, 1997.

Mike Develin, American Institute of Mathematics, 360 Portage Ave., Palo Alto, CA 94306-2244, USA

E-mail address: develin@post.harvard.edu

Jeremy L. Martin, Department of Mathematics, University of Kansas, Lawrence, KS 66045, USA

E-mail address: jmartin@math.ku.edu

Victor Reiner, School of Mathematics, University of Minnesota, Minneapolis, MN 55455, USA

E-mail address: reiner@math.umn.edu 